\title{
Design, synthesis, anti-tumor activity, and molecular modeling of quinazoline and pyrido[2,3- $d]$ pyrimidine derivatives targeting epidermal growth factor receptor
}

$\mathrm{Ju} \mathrm{Hou}^{1}$, Shanhe Wan ${ }^{1}$, Guangfa Wang, Tingting Zhang, Zhonghuang Li, Yuanxin Tian, Yonghuan $\mathrm{Yu}$, Xiaoyun $\mathrm{Wu}^{* *}$, Jiajie Zhang*

Guangdong Provincial Key Laboratory of New Drug Screening, School of Pharmaceutical Science, Southern Medical University, Guangzhou 510515, PR China.

*Corresponding author. Tel.: +86 20 62789415; fax:+86 2061648548.

*** Corresponding author. Tel.: +86 2062789416.

E-mail addresses: xywugz@163.com (X.-Y.Wu), zhangjj@smu.edu.cn(J.-J. Zhang).

${ }^{1}$ These authors contributed equally to this work.

Abstract: Three series of novel quinazoline and pyrido[2,3- $d$ ]pyrimidine derivatives were designed, synthesized and evaluated for their ability to inhibit EGFR tyrosine kinase and a panel of five human cancer cell lines (MCF-7, A549, BT-474, SK-BR-3, and MDA-MB-231). Bioassay results indicated that five of these prepared compounds (12c-12e and 13c-13d) exhibited remarkably higher inhibitory activities against EGFR and SK-BR-3 cell line. Compounds 12c and 12e displayed the most potent EGFR inhibitory activity ( $\mathrm{IC}_{50}=2.97 \mathrm{nM}$ and $3.58 \mathrm{nM}$, respectively) and good anti-proliferative effect against SK-BR-3 cell with the $\mathrm{IC}_{50}$ values of $3.10 \mu \mathrm{M}$ and $5.87 \mu \mathrm{M}$, respectively. Furthermore, molecular docking and molecular dynamics simulation studies verified that compound $\mathbf{1 2} \mathrm{c}$ and $\mathbf{1 2} \mathrm{e}$ shared similar binding pattern with gefitinib in the binding pocket of EGFR. MM-GBSA binding free energy revealed that the compound $12 \mathrm{c}$ and $\mathbf{1 2} \mathrm{e}$ have almost the same inhibitory activity against EGFR as gefitinib, and that the dominating effect of van der Waals interactions drives the binding process.

Keywords: EGFR inhibitor; Quinazoline and pyrido[2,3- $d$ ]pyrimidine derivatives; Antitumor; Molecular docking; Molecular dynamics simulation.

\section{Introduction}

Epidermal growth factor receptor (EGFR) belongs to the ErbB family of receptor tyrosine kinases. This family includes homologous receptors: the epidermal growth factor receptor (ErbB1/EGFr/HER1), ErbB2 (HER2/neu), ErbB3 (HER3), and ErbB4 
(HER4). These receptors are composed of an extracellular ligand-binding domain, a transmembrane lipophilic segment, and an intracellular protein tyrosine kinase domain with a regulatory carboxyl terminal segment [1]. When ligands bind to these domains, receptor dimerization and autophosphorylation of intracellular tyrosine kinase domains occur. Autophosphorylation activating the downstream signaling pathways leads to diverse effects including cell proliferation, survival, adhesion, migration, and differentiation $[2,3]$. Previous research has demonstrated that EGFR is a putative oncogene and has been found in many human solid malignancies including non-small-cell lung cancer, ovarian cancer, breast cancer, etc [4] . Therefore, EGFR tyrosine kinase represents an attractive target for the treatment of many human cancers.

Quinazoline derivatives have long been recognized as an important scaffold for designing anti-EGFR agents $[5,6]$. The clinical success of selective kinase inhibitors, such as gefitinib [7, 8], lapatinib [9] , erlotinib [10], and canertinib [11, 12] (Fig. 1), as therapeutic agents for several human cancers has prompted substantial interest in the further development and clinical testing of such inhibitors for a wide variety of malignancies. On the other hand, pyrido[2,3-d]pyrimidine derivatives also can be interacted with ATP-binding site of EGFR catalytic domain to inhibit EGFR [13, 14]. Compound A-1 (Fig. 1) belongs to the pyrido[2,3-d]pyrimidines, which showed nanomolarly potent EGFR inhibitory activity. Quinazoline and pyrido[2,3-d]pyrimidine derivatives displayed favorable inhibitory activities against EGFR spured us on to greater efforts to develop novel and highly potent EGFR inhibitors.

Based on the crystal structure of EGFR complexed with gefitinib, we undertook the design of quinazoline and pyrido[2,3-d]pyrimidine derivatives having different substitutents at C-2, C-4, and C-6 positions. To increase the hydrogen bond interactions with hinge region of the kinase, an amino group was introduced to $\mathrm{C}-2$ position of the quinazoline core. In order to extend into the solvent region surrounding the kinase hinge segment, introduction of the polar N-substituted pyrazole fragments at the C-6 position of quinazoline and pyrido[2,3-d]pyrimidine scaffolds was investigated. Different kinds of aryl fragments connected to quinazoline and pyrido[2,3- $d$ ]pyrimidine nucleus through O-linker or N-linker at $\mathrm{C}-4$ position were investigated to increase hydrophobic interactions with the hydrophobic pocket, which are crucial for EGFR inhibitory activity. Herein, we would like to report the synthesis and biological evaluation of these new series compounds for the first time. 


\section{Results and discussion}

\subsection{Chemistry}

The synthetic strategy to prepare the target compounds is illustrated in Schemes 1-3. The bromination of the starting material 1 led to 2-amino-5-bromonicotinic acid (2), followed by cycilzation with formamide to give compound 3 [15], which was chloride with thionyl chloride using DMF as catalyst to afford 4 . The chlorine in the 4-position of intermediate 4 could be displaced with a variety of benzylamines to achieve intermediates 5a-5d. Introduction of the pyrazole fragment at the C-6 position of 5a-5d by conventional Suzuki coupling with the corresponding pyrazole boronic esters to obtain desired compounds $\mathbf{7 a - 7 d}$ of series-I, or further deprotection of the Boc group of intermediate smoothly to give desired compound $\mathbf{6}$ of series-I [15].

Likewise, compounds 9 and 10, which were prepared following the similar procedure described above for the preparation of $\mathbf{3}$ and $\mathbf{4}$, respectively. Compound $\mathbf{1 0}$ was displaced with a variety of nucleophiles. The resulting intermediates 11a-11g were converted to target compounds 12a-12f and 13a-13d of series-II by Suzuki coupling reaction as described in Scheme 2.

The intermediate 16 was produced from 14 by the reaction with dicyandiamide followed by the cleavage of the amidino moiety of 15 [16], which was condensed with different reagents to give the desired products 17a-17b. Final compounds 18 and 19a-19b of series-II were similarly obtained of series-I as shown in Scheme 3.

The structures of all the newly synthesized compounds were characterized by ${ }^{1} \mathrm{H}$ NMR, ${ }^{13} \mathrm{C}$ NMR, ESI-MS, and HRMS.

\subsection{Biological activity}

\subsubsection{Kinase inhibitory activity}

The enzyme activity assays of new compounds were performed at a single-point concentration $(1 \mu \mathrm{M})$ against EGFR and their inhibitory activities were displayed at Table 1. As shown in Table 1, compounds 12c-12e and 13c-13d exhibited favorable inhibition of the ErbB1 (percent inhibition values range of 99-102\%), while compounds 7d, 12a, 12f, 13a, and 13b showed moderate inhibitory activities (percentage of inhibition raning from $30 \%$ to $67 \%$ ) and others had no obvious 
inhibitory activities.

Subsequently, we further evaluated the EGFR inhibitory activities of compounds 12c-12e and 13c-13d with excellent inhibition at $1 \mu \mathrm{M}$. As shown in Table 2, the $\mathrm{IC}_{50}$ values ranging from 2.97 to $108 \mathrm{nM}$ against ErbB1 and EGFR (L858R). Compounds 12c-12e and 13c-13d were chosen for further evaluation of the selectivity of EGFR kinase, the $\mathrm{IC}_{50}$ values of $12 \mathrm{c}-12 \mathrm{e}$ and $\mathbf{1 3 c - 1 3 d}$ ranging from 590 to $1603 \mathrm{nM}$ against HER2, and HER4, while compounds 12c-12e and 13c-13d had no obvious inhibitory activity toward EGFR (T790M/L858R). These results indicated that compounds 12c-12e and 13c-13d had good selectivity to EGFR kinase.

\subsubsection{In vitro anti-proliferative activity}

The antiproliferative activities of newly synthesized compounds were evaluated against a panel of five human cancer cell lines overexpressing EGFR, including MCF-7, A549, BT-474, SK-BR-3, and MDA-MB-231 by the standard MTT assay in vitro [18-20], with gefitinib as the positive control. The inhibitory activities $\left(\mathrm{IC}_{50}\right)$ were summarized in Table 1. As depicted in Table 1, the results indicated that most compounds showed moderate to good activity with $\mathrm{IC}_{50}$ values in the $\mu \mathrm{M}$ range, while compounds $\mathbf{7 a - 7 d}$ proved to be ineffective against the five cell lines, and compounds $12 \mathrm{c}$ and 18 also showed no inhibitory activity against $\mathrm{A} 549$ cell line $\left(\mathrm{IC}_{50}>100 \mu \mathrm{M}\right)$. Some of the synthesized compounds exhibited similar anti-proliferative activities as gefitinib against the three to four cell lines. Specifically compounds $\mathbf{6}, \mathbf{1 2 d}, \mathbf{1 3 a}-\mathbf{1 3 b}$, 18, and 19a-19b showed higher antiproliferative activities than gefitinib against MCF-7 cell line. In addition, five compounds (12c-12e and 13c-13d) exhibited remarkable inhibitory activity with the $\mathrm{IC}_{50}$ values ranging from 0.064 to $5.87 \mu \mathrm{M}$, which were comparable to that of gefitinib $\left(\mathrm{IC}_{50}=4.92 \mu \mathrm{M}\right)$ on SK-BR-3 cell line. All the compounds displayed poor activities $\left(\mathrm{IC}_{50}: 3.00->100 \mu \mathrm{M}\right)$ in BT-474 cancer cell line, as compared with gefitinib.

This primary screening revealed that 4-aminoquinazoline core derivatives exhibited strong EGFR enzymatic potency and anti-proliferative activities. Specifically aniline moieties and quinoline amino fragment at C-4 position of the quinazoline core showed promising inhibitory activity against EGFR and potent activities toward SK-BR-3 cell line, and had the makings of good drugs for breast cancer. The pyrido[2,3- $d]$ pyrimidine derivatives dispalyed a significant loss in potency compared to that of 4-aminoquinazoline core derivatives. Moreover, 
2-aminoquinazoline nucleus derivatives gave weak EGFR inhibitory activities. The enzymatic and cellular responses were inconsistent for some target compounds. Notably, compounds 6, 13a-13b, 18, and 19a-19b displayed poor activities against EGFR kinase, but they showed simliar or higher antiproliferative activities than gefitinib against some cancer cell lines that express high levels of EGFR. It suggested that the compounds might induce cells apoptosis, or accelerate cell metabolism, or have some other mechanisms.

\subsection{Preliminary structure-activity relationships}

Based on biological activity evaluation of the synthetic compounds, the structure-activity relationships of these novel compounds were primarily analyzed. As shown in Fig. 2, the pyrido[2,3- $d$ ]pyrimidine derivatives show decreased inhibitory activity, and introduction of amino group at C-2 position of the quinazoline core did not led to significant inhibitory activity. Moreover, N-linker compounds showed good inhibitory activity than O-linker at C-4 position of scaffolds; aniline moieties directly conjunct to the 4-position of quinazoline core increased EGFR inhibitory activities as compared to benzyl amino groups. Interestingly, quinoline amino fragment at C-4 position of the quinazoline core also exhibited strong EGFR enzymatic and cellular potency.

\subsection{Molecular modeling}

To predict the possible binding mode of designed compounds with EGFR, a study of docking of all compounds into the active site of the EGFR (PDB ID: 4WKQ) were performed using Surflex-Dock and then molecular dynamics (MD) simulations were performed by using AMBER software package.

The docking reliability was validated using the known X-ray structure of EGFR complexed with gefitinib. The co-crystallized gefitinib was re-docked into the binding site, and the docked conformation with the highest total score was selected as the most probable binding conformation. As shown in Fig. 3, the redocked gefitinib is almost in the same position with co-crystallized gefitinib at the active site of EGFR except the flexible long chain extending into the solvent accessible region, which have revealed that the high reliability of Surflex-dock in reproducing the experimentally observed binding mode for the EGFR inhibitors. Therefore, Surflex-Dock method was used in search of the binding conformations of the newly synthesised compounds. All compounds, except 2-amino-quinazoline dereivatives 18 
and 19a-19b, were successfully docked into the active site of EGFR (Fig. 4). As is shown in Fig. 4, quinazoline and pyrido[2,3-d]pyrimidine compounds share similar binding pattern in the binding pocket with the co-crystal ligand gefitinib in the reference crystal structure, while the 2-amino-quinazoline dereivatives is shifted out of the binding pocket to the solvent surface, which may be the reason of the low potency of 2-amino-quinazoline compounds.

MD simulations were carried out on active inhibitors 12c and 12e in complex with EGFR to explore in depth the binding poses. For comparison, the MD simulations of positive control gefitinib and less active compound $7 \mathbf{a}$ complexed with EGFR were also carried out.

MD simulations were processed in explicit aqueous solution for $20 \mathrm{~ns}$. The stability of the system under simulation was evaluated by the root-mean-square deviation (RMSD) of the backbone atoms relate to the starting structures (Fig. 5). As can be seen in the plot, all systems reached equilibrium after $8 \mathrm{~ns}$ simulation times. Fig. 6 shows the binding poses of the two active compounds $12 \mathrm{c}$ and 12e in the binding pocket of EGFR. As illustrated in Fig. 6, the binding modes of 12c and 12e from MD simulated results are nearly the same as the docked structures. The quinazoline moiety inserts into deep hydrophobic pocket, the aniline moiety is deeply in the back of the ATP-binding pocket. The pyrazole substituent occupies the solvent exposed region, indicating that the introduction of hydrophilic groups here probably is beneficial for potency.

The hydrogen bond plays an important role in inhibitor binding to kinase [22]. The hydrogen-bond interactions were examined and found that N1 of quinazoline ring of gefitinib, 12c and 12e establish strong hydrogen interaction with the backbone $\mathrm{NH}$ of Met-793 in the hinge region of EGFR during all the MD simulation time (Table 3), while the key hydrogen-bond interaction could not be formed in the system of 7a-EGFR, which partically explains the low potency of compound 7a.

The binding free energies were calculated by using MM-GBSA program in AMBER taking the last 10ns of the MD simulation. In Table 4, we present the predicted binding free energies, together with their respective enthalpic and entropic contributions. As can been seen, MM-GBSA calculations verified that ligands $\mathbf{1 2 c}$ and 12e have almost the same activities against EGFR as the positive control gefitinib, which is in good agreement with the experimental data. In addition, the binding free energy of compound 7a binding to EGFR is approximately $3 \mathrm{kcal} / \mathrm{mol}$ more than that 
of gefitinib, in consistent with the experimental observation that ligand 7a exhibits drastically diminished potency.

According to the energy individual components of the binding free energies, the favorable contributors to ligand binding are van der Waals (vdW) terms, electrostatic and nonpolar salvation energies, whereas polar solvation and entropy terms oppose binding. The favorable electrostatic interactions are counteracted by the unfavorable electrostatics of desolvation upon binding. Consequently, the total electrostatic interaction contributions are unfavorable to binding in all systems. The vdW contribution interaction upon binding is very important to the binding of inhibitors with EGFR. Comparing 7a-EGFR with the other complexs, the most important terms which dictates the difference in the binding affinity are $\Delta \mathrm{G}_{\mathrm{ele}}$ and $\Delta \mathrm{G}_{\mathrm{vdW}}$.

\section{Conclusion}

In summary, three series of novel quinazoline and pyrido[2,3- $d$ ]pyrimidine derivatives were designed, synthesized and screened for biological activity as novel EGFR inhibitors. The preliminary investigation shows some compounds displayed favorable effect in the EGFR kinase assay and antiproliferative assay. Among them, compounds 12c and 12e showed the higher potency to EGFR kinase. In addition, the molecular docking and MD simulations experiments have revealed that the binding poses of 12c and 12e with EGFR are similar to that of gefitinib. MM-GBSA binding free energy revealed that the compound $12 \mathrm{c}$ and $12 \mathrm{e}$ have almost the same inhibitory activity against EGFR as gefitinib, and that the dominating effect of vdW interactions drives the binding process. Compounds $12 \mathrm{c}$ and $12 \mathrm{e}$ could be worth of further development, and these results expand the chemical diversity of EGFR inhibitors.

\section{Experimental}

\subsection{Chemistry}

All reagents were purchased from commercial vendors and were used without further purification. All oxygen-sensitive or moisture-sensitive reactions were run under nitrogen atmosphere. ${ }^{1} \mathrm{H}$ and ${ }^{13} \mathrm{C}$ NMR spectra were recorded on a BRUKER AVIII $400 \mathrm{MHz}$ and $101 \mathrm{MHz}$ spectrometer with tetramethylsilane (TMS) as the internal standard, the values of the chemical shifts $(\delta)$ are given in ppm, and coupling constants $(J)$ are given in Hz. Mass spectra (ESI-MS) were performed on WATERS ZQ4000. Highresolution mass spectra (HRMS) were recorded on AB Sciex 
TripleTOF 5600 mass spectrometer. All reactions were monitored using thin-layer chromatography (TLC) on silica gel plates at $254 \mathrm{~nm}$ under a UV laMp. Flach column chromatography separations were performed on normal phase silica gel (200-300 mesh, Merck) or reverse phase silica gel by using Yamazen AI-580 flash chromatography with UV detection at $254 \mathrm{~nm}$. Melting points were determined using a X-4 Melting-point Apparatus with Microscope (Gongyi City Yuhua Instrument Co., Ltd., Hennan, China) and were uncorrected.

\section{1-(2,6-Dichloro-3-fluorophenyl)ethan-1-amine [15]}

Preparation of 1-(2,6-Dichloro-3-fluorophenyl)ethan-1-amine and characterization data have already been reported. ${ }^{1} \mathrm{H}$ NMR $\left(400 \mathrm{MHz}, \mathrm{DMSO}-d_{6}\right) \delta 8.72(\mathrm{~s}, 2 \mathrm{H})$, $7.66(\mathrm{dd}, J=5.2,9.2 \mathrm{~Hz}, 1 \mathrm{H}), 7.58(\mathrm{t}, J=8.8 \mathrm{~Hz}, 1 \mathrm{H}), 5.03(\mathrm{q}, J=6.8 \mathrm{~Hz}, 1 \mathrm{H}), 1.63$ $(\mathrm{d}, J=7.2 \mathrm{~Hz}, 1 \mathrm{H}), \mathrm{ESI}-\mathrm{MS} \mathrm{m} / \mathrm{z}: 208.0[\mathrm{M}+\mathrm{H}]^{+}$.

1-(pyridin-3-yl)ethanamine [17]

Preparation of 1-(pyridin-3-yl)ethanamine and characterization data have already been reported. ${ }^{1} \mathrm{H}$ NMR (400 MHz, MeOD) $\delta 8.48(\mathrm{~d}, J=6.4 \mathrm{~Hz}, 2 \mathrm{H}), 7.46(\mathrm{~d}, J=$ $6.0 \mathrm{~Hz}, 2 \mathrm{H}), 4.08(\mathrm{q}, J=6.6 \mathrm{~Hz}, 1 \mathrm{H}), 1.41(\mathrm{~d}, J=6.8 \mathrm{~Hz}, 3 \mathrm{H})$, ESI-MS m/z: 123.2 $[\mathrm{M}+\mathrm{H}]^{+}$.

\section{2-amino-5-bromonicotinic acid (2)}

Preparation of 2 and characterization data have already been reported. ${ }^{1} \mathrm{H}$ NMR (400 MHz, MeOD) $\delta 9.24(\mathrm{~d}, J=2.0 \mathrm{~Hz}, 1 \mathrm{H}), 9.14$ (d, $J=2.0 \mathrm{~Hz}, 1 \mathrm{H})$. ESI-MS m/z: $216.7[\mathrm{M}+\mathrm{H}]^{+}$.

6-bromopyrido[2,3-d]pyrimidin-4-ol (3)

2-amino-5-bromonicotinic acid (2) (21.6 g, $0.10 \mathrm{~mol})$ was added to a solution of formamide $100 \mathrm{~mL}$, and then the reaction mixture was stirred at reflux for $5 \mathrm{~h}$. The mixture was poured into $800 \mathrm{~mL} \mathrm{H}_{2} \mathrm{O}$. The resulting precipitate was filtered, washed with water, and dried to give 3 as an off-white solid (16.9 g, yield, 74\%). ${ }^{1} \mathrm{H}$ NMR (400 MHz, DMSO- $\left.d_{6}\right) \delta 12.72(\mathrm{~s}, 1 \mathrm{H}), 9.05(\mathrm{~d}, J=2.4 \mathrm{~Hz}, 1 \mathrm{H}), 8.63(\mathrm{~d}, J=2.4 \mathrm{~Hz}$, 1H), 8.36 (s, 1H). ESI-MS m/z: $228.3[\mathrm{M}+\mathrm{H}]^{+}$.

\section{6-bromo-4-chloropyrido[2,3-d]pyrimidine (4)}

To the suspension of 6-bromopyrido[2,3-d]pyrimidin-4-ol (3) (15.0 g, $0.066 \mathrm{~mol}$ ) in thionyl chloride $150 \mathrm{~mL}$ was added a few drops of DMF, then the mixture was 
stirred at reflux for $6 \mathrm{~h}$. After removing thionyl chloride under vacuum, the residue was recrystallized from EtOAc to gave 4 as pale yellow solid $(11.8 \mathrm{~g}$, yield, $73 \%) .{ }^{1} \mathrm{H}$ NMR (400 MHz, $\left.\mathrm{CDCl}_{3}\right) \delta 9.33(\mathrm{~d}, J=7.6 \mathrm{~Hz}, 2 \mathrm{H}), 8.80$ (s, 1H). ESI-MS m/z: 246.9 $[\mathrm{M}+\mathrm{H}]^{+}$.

6-bromo-N-(1-(2,6-dichloro-3-fluorophenyl)ethyl)pyrido[2,3-d]pyrimidin-4-amine (5a)

To a solution of compound $4(2.43 \mathrm{~g}, 0.01 \mathrm{~mol})$ in $25 \mathrm{~mL}$ dioxane was added 1-(2,6-Dichloro-3-fluorophenyl)ethan-1-amine (3.12 g, $0.015 \mathrm{~mol})$, and then the reaction mixture was stirred at room temperature overnight. After that, the mixture was poured into $150 \mathrm{~mL} \mathrm{H}_{2} \mathrm{O}$, and then the resulting precipitate was filtered, washed with 50\% ethanol, and dried overnight under vacuum to give 5a as white solid (3.58 g, yield, 86\%). ${ }^{1} \mathrm{H}$ NMR (400 MHz, DMSO- $d_{6}$ ) $\delta 9.40$ (d, $\left.J=2.4 \mathrm{~Hz}, 1 \mathrm{H}\right), 9.07$ (d, $J=$ $2.0 \mathrm{~Hz}, 1 \mathrm{H}), 9.03$ (d, $J=5.2 \mathrm{~Hz}, 1 \mathrm{H}), 8.48(\mathrm{~s}, 1 \mathrm{H}), 7.47-7.44(\mathrm{~m}, 1 \mathrm{H}), 7.35$ (t, $J=$ $8.8 \mathrm{~Hz}, 1 \mathrm{H}), 5.85$ (q, $J=7.0 \mathrm{~Hz}, 1 \mathrm{H}), 1.71$ (d, $J=7.2 \mathrm{~Hz}, 3 \mathrm{H})$. ESI-MS m/z: 417.4 $[\mathrm{M}+\mathrm{H}]^{+}$.

\section{6-bromo-N-(1-(pyridin-4-yl)ethyl)pyrido[2,3-d]pyrimidin-4-amine (5b)}

Compound $\mathbf{5 b}$ was synthesized from 4 and 1-(pyridin-3-yl)ethanamine following the similar procedure described above for the preparation of $\mathbf{5 a}$ (yield, 76\%). ${ }^{1} \mathrm{H}$ NMR $\left(400 \mathrm{MHz}, \mathrm{DMSO}-d_{6}\right) \delta 9.27(\mathrm{~d}, J=2.4 \mathrm{~Hz}, 1 \mathrm{H}), 9.08(\mathrm{~d}, J=2.4 \mathrm{~Hz}, 1 \mathrm{H}), 8.90(\mathrm{~d}, J$ $=7.4 \mathrm{~Hz}, 1 \mathrm{H}), 8.57(\mathrm{~s}, 1 \mathrm{H}), 8.50(\mathrm{dd}, J=1.6,4.8 \mathrm{~Hz}, 2 \mathrm{H}), 7.43(\mathrm{dd}, J=1.2,4.4 \mathrm{~Hz}$, $2 \mathrm{H}), 5.50(\mathrm{q}, J=7.0 \mathrm{~Hz}, 1 \mathrm{H}), 1.60(\mathrm{~d}, J=7.2 \mathrm{~Hz}, 3 \mathrm{H})$. ESI-MS m/z: $332.5[\mathrm{M}+\mathrm{H}]^{+}$.

\section{6-bromo-N-(3-methoxybenzyl)pyrido[2,3-d]pyrimidin-4-amine (5c)}

Compound 5c was synthesized from 4 and (3-methoxyphenyl)methanamine following the similar procedure described above for the preparation of 5a (yield, $58 \%$ ). ${ }^{1} \mathrm{H}$ NMR (400 MHz, DMSO- $\left.d_{6}\right) \delta 9.16(\mathrm{t}, J=5.6 \mathrm{~Hz}, 1 \mathrm{H}), 9.11(\mathrm{~d}, J=2.4 \mathrm{~Hz}$, $1 \mathrm{H}), 9.07(\mathrm{~d}, J=2.4 \mathrm{~Hz}, 1 \mathrm{H}), 8.64(\mathrm{~s}, 1 \mathrm{H}), 7.25(\mathrm{t}, J=8.2 \mathrm{~Hz}, 1 \mathrm{H}), 6.96-6.95(\mathrm{~m}$, 2H), $6.86-6.85(\mathrm{~m}, 1 \mathrm{H}), 4.77$ (d, $J=5.6 \mathrm{~Hz}, 2 \mathrm{H}), 3.74$ (s, 3H). ESI-MS m/z: 347.1 $[\mathrm{M}+\mathrm{H}]^{+}$.

\section{6-bromo-N-(3-nitrobenzyl)pyrido[2,3-d]pyrimidin-4-amine (5d)}

Compound 5d was synthesized from $\mathbf{4}$ and (3-nitrophenyl)methanamine following the similar procedure described above for the preparation of 5a (yield, 72\%). ${ }^{1} \mathrm{H}$ NMR (400 MHz, DMSO- $\left.d_{6}\right) \delta 9.66(\mathrm{~s}, 1 \mathrm{H}), 9.14(\mathrm{~d}, J=8.0 \mathrm{~Hz}, 2 \mathrm{H}), 8.72(\mathrm{~s}$, 
1H), $8.28(\mathrm{~s}, 1 \mathrm{H}), 8.15(\mathrm{~d}, J=7.6 \mathrm{~Hz}, 1 \mathrm{H}), 7.88(\mathrm{~d}, J=7.6 \mathrm{~Hz}, 1 \mathrm{H}), 7.65(\mathrm{t}, J=7.8$ $\mathrm{Hz}, 1 \mathrm{H}), 4.95(\mathrm{~d}, J=5.2 \mathrm{~Hz}, 2 \mathrm{H})$. ESI-MS m/z: $362.3[\mathrm{M}+\mathrm{H}]^{+}$.

N-(1-(2,6-dichloro-3-fluorophenyl)ethyl)-6-(1-(piperidin-4-yl)-1H-pyrazol-4-yl)pyrid o[2,3-d]pyrimidin-4-amine (6)

A mixture of compound 5a (457 mg, $1.1 \mathrm{mmol})$, tert-Butyl 4-[4-(4,4,5,5-tetramethyl-1,3,2-dioxaborolan-2-yl)-1H-pyrazol-1-yl]piperidine-1-carboxylate (21) (400 $\mathrm{mg}, 1.06 \mathrm{mmol}), \mathrm{PdCl}_{2}$ (dppf) $\mathrm{CH}_{2} \mathrm{Cl}_{2}(80.2 \mathrm{mg}, 0.098 \mathrm{mmol})$, and $\mathrm{K}_{2} \mathrm{CO}_{3}(420 \mathrm{mg}$, $3.04 \mathrm{mmol})$ in dioxane : $\mathrm{H}_{2} \mathrm{O}(24: 1)(12 \mathrm{~mL})$ was degassed and charged with nitrogen for three times and then heated in an oil bath at $80{ }^{\circ} \mathrm{C}$ for $4 \mathrm{~h}$ under the protection of nitrogen. The reaction mixture was diluted with water $(30 \mathrm{~mL})$ and eracted with EtOAc $(30 \mathrm{~mL} \times 3)$. The combined organic layer was dried over anhydrous $\mathrm{Na}_{2} \mathrm{SO}_{4}$, filtered, and concentrated. The concentrates was purified by flash chromatography on silica gel eluting with petroleum ether-EtOAc-methnaol (containing 1\% ammonia) (40:60:0.05) to give a compound as a white solid. The solid was dissolved in a mixed solvent system $\left(\mathrm{CH}_{2} \mathrm{Cl}_{2} / \mathrm{TFA}=4: 1,20 \mathrm{~mL}\right)$, then the reaction mixture was stirred at room temperature overnight, and concentrated. The residue was triturated with acetone-petroleum ether (1:1) to give 6 as a white solid (231 mg, yield, 48\%). mp 265-267 ${ }^{\circ} \mathrm{C} .{ }^{1} \mathrm{H}$ NMR (400 MHz, DMSO-d $\left.d_{6}\right) \delta 11.22$ (s, 1H), 10.13 (s, 1H), 9.42 (s, 1H), $9.21(\mathrm{~s}, 1 \mathrm{H}), 8.78(\mathrm{~s}, 2 \mathrm{H}), 8.39(\mathrm{~s}, 1 \mathrm{H}), 7.49(\mathrm{~s}, 1 \mathrm{H}), 7.39(\mathrm{t}, J=7.2 \mathrm{~Hz}, 1 \mathrm{H}) 5.96$ (s, 1H), 4.60 (s, 1H), 3.61(s, 2H), $3.12(\mathrm{~s}, 2 \mathrm{H}), 2.27$ (s, 4H), 1.87 (d, J=6.0 Hz, 3H). ${ }^{13} \mathrm{C}$ NMR (101 MHz, MeOD) $\delta 161.0,158.5,156.0,155.1,151.8,146.4,138.1,137.1$, 130.4, 129.9, 128.1, 127.6, 117.6, 116.0, 115.8, 109.2, 56.1, 51.3, 43.0, 28.9, 16.1 . ESI-MS m/z: $486.5[\mathrm{M}+\mathrm{H}]^{+}$. HRMS, $\mathrm{ESI}^{+}, \mathrm{m} / \mathrm{z}$ : Calcd for $\mathrm{C}_{23} \mathrm{H}_{22} \mathrm{Cl}_{2} \mathrm{FN}_{7}(\mathrm{M}+\mathrm{H})^{+}$, 486.1371; found, 486.1368 .

N-(1-(2,6-dichloro-3-fluorophenyl)ethyl)-6-(1-methyl-1H-pyrazol-4-yl)pyrido[2,3-d]p yrimidin-4-amine (7a)

A mixture of compound $\mathbf{5 a}(208 \mathrm{mg}, 0.50 \mathrm{mmol})$, 1-methyl-4-(4,4,5,5-tetramethyl-1,3,2-dioxaborolan-2-yl)-1H-pyrazole (20) (108 mg, $0.52 \mathrm{mmol}$ ), $\mathrm{PdCl}_{2}$ (dppf)$\mathrm{CH}_{2} \mathrm{Cl}_{2}(40.1 \mathrm{mg}, 0.05 \mathrm{mmol})$ and $\mathrm{K}_{2} \mathrm{CO}_{3}(207 \mathrm{mg}, 1.5 \mathrm{mmol})$ in dioxane $: \mathrm{H}_{2} \mathrm{O}$ $(24: 1)(15 \mathrm{~mL})$ was degassed and charged with nitrogen for three times and then heated in an oil bath at $80{ }^{\circ} \mathrm{C}$ for $4 \mathrm{~h}$ under the protection of nitrogen. The reaction mixture was diluted with water $(30 \mathrm{~mL})$ and eracted with EtOAc $(30 \mathrm{~mL} \times 3)$. The combined organic layer was dried over anhydrous $\mathrm{Na}_{2} \mathrm{SO}_{4}$, filtered, and concentrated. 
The concentrates was purified by flash chromatography on silica gel eluting with petroleum ether-EtOAc-methnaol (containing 1\% ammonia) (40:60:0.05) to give 7a (129 mg, yield, 62\%). mp 268-271 ${ }^{\circ} \mathrm{C} .{ }^{1} \mathrm{H}$ NMR (400 MHz, MeOD) $\delta 9.20$ (d, $J=2.0$ Hz, 1H), 9.06 (d, $J=2.4 \mathrm{~Hz}, 1 \mathrm{H}), 8.43$ (s, 1H), 8.21 (s, 1H), 8.07 (s, 1H), 7.35 (dd, $J$ $=5.2,8.8 \mathrm{~Hz}, 1 \mathrm{H}), 7.14(\mathrm{t}, J=8.6 \mathrm{~Hz}, 1 \mathrm{H}), 6.07(\mathrm{q}, J=7.4 \mathrm{~Hz}, 1 \mathrm{H}), 4.01(\mathrm{~s}, 3 \mathrm{H})$, $1.83(\mathrm{~d}, J=7.2 \mathrm{~Hz}, 3 \mathrm{H}) .{ }^{13} \mathrm{C} \mathrm{NMR}(101 \mathrm{MHz}, \mathrm{MeOD}) \delta 159.6,157.2,155.9,152.9$, 139.6, 136.5, 129.5, 129.4, 128.4, 127.1, 126.9, 118.8, 115.2, 114.9, 109.6, 49.3, 37.8, 16.3. ESI-MS m/z: $417.5[\mathrm{M}+\mathrm{H}]^{+}$. HRMS, ESI ${ }^{+}, \mathrm{m} / \mathrm{z}$ : Calcd for $\mathrm{C}_{19} \mathrm{H}_{15} \mathrm{Cl}_{2} \mathrm{FN}_{6}$ $(\mathrm{M}+\mathrm{H})^{+}, 417.0792$; found, 417.0794 .

6-(1-methyl-1H-pyrazol-4-yl)-N-(1-(pyridin-4-yl)ethyl)pyrido[2,3-d]pyrimidin-4-ami$n e(7 \mathbf{b})$

Compound $\mathbf{7 b}$ was synthesized from $\mathbf{5 b}$ and $\mathbf{2 0}$ following the similar procedure described above for the preparation of 7a (yield, 55\%). mp 152-154 ${ }^{\circ} \mathrm{C} .{ }^{1} \mathrm{H}$ NMR (400 MHz, MeOD) $\delta 9.20(\mathrm{~d}, J=9.6 \mathrm{~Hz}, 1 \mathrm{H}), 8.99(\mathrm{~s}, 1 \mathrm{H}), 8.49(\mathrm{~d}, J=4.8 \mathrm{~Hz}, 3 \mathrm{H}), 8.20$ $(\mathrm{d}, J=7.6 \mathrm{~Hz}, 1 \mathrm{H}), 8.05(\mathrm{~d}, J=7.2 \mathrm{~Hz}, 1 \mathrm{H}), 7.52(\mathrm{~d}, J=5.6 \mathrm{~Hz}, 2 \mathrm{H}), 5.61(\mathrm{q}, J=5.6$ $\mathrm{Hz}, 1 \mathrm{H}), 4.00$ (s, 3H), 1.74 (d, $J=7.2 \mathrm{~Hz}, 3 \mathrm{H}) .{ }^{13} \mathrm{C} \mathrm{NMR}(101 \mathrm{MHz}, \mathrm{MeOD}) \delta 160.3$, 157.1 , 156.0, 154.4, 153.1, 148.8, 136.5, 128.4, 127.1, 126.9, 121.7, 118.7, 109.8, 49.9, 37.8, 20.3. ESI-MS m/z: $332.6[\mathrm{M}+\mathrm{H}]^{+}$. HRMS, ESI ${ }^{+}, \mathrm{m} / \mathrm{z}$ : Calcd for $\mathrm{C}_{18} \mathrm{H}_{17} \mathrm{~N}_{7}$ $(\mathrm{M}+\mathrm{H})^{+}, 332.1618$; found, 332.1621 .

N-(3-methoxybenzyl)-6-(1-methyl-1H-pyrazol-4-yl)pyrido[2,3-d]pyrimidin-4-amine (7c)

Compound 7c was synthesized from $\mathbf{5 c}$ and 20 following the similar procedure described above for the preparation of 7a (yield, 67\%). mp 196-198 ${ }^{\circ} \mathrm{C} .{ }^{1} \mathrm{H}$ NMR (400 MHz, DMSO- $\left.d_{6}\right) \delta 9.28(\mathrm{~s}, 1 \mathrm{H}), 9.11(\mathrm{~s}, 1 \mathrm{H}), 8.99(\mathrm{~s}, 1 \mathrm{H}), 8.58(\mathrm{~s}, 1 \mathrm{H}), 8.32(\mathrm{~s}, 1 \mathrm{H})$, $8.03(\mathrm{~s}, 1 \mathrm{H}), 7.26(\mathrm{t}, J=8.0 \mathrm{~Hz}, 1 \mathrm{H}), 6.98(\mathrm{~s}, 2 \mathrm{H}), 6.85(\mathrm{~d}, J=7.6 \mathrm{~Hz}, 1 \mathrm{H}), 4.82(\mathrm{~d}, J$ $=5.6 \mathrm{~Hz}, 2 \mathrm{H}), 3.92(\mathrm{~s}, 3 \mathrm{H}), 3.74(\mathrm{~s}, 3 \mathrm{H}) .{ }^{13} \mathrm{C} \mathrm{NMR}\left(101 \mathrm{MHz}, \mathrm{DMSO}-d_{6}\right) \delta 159.8$, 159.6, 154.8, 148.1, 141.5, 136.7, 130.6, 130.6, 129.9, 128.5, 128.4, 121.9, 119.8, 117.9, 115.7, 113.6, 112.5, 55.4, 43.9, 39.2. ESI-MS m/z: $347.4[\mathrm{M}+\mathrm{H}]^{+} . \mathrm{HRMS} \mathrm{ESI}^{+}$, $\mathrm{m} / \mathrm{z}$ : Calcd for $\mathrm{C}_{19} \mathrm{H}_{18} \mathrm{~N}_{6} \mathrm{O}(\mathrm{M}+\mathrm{H})^{+}, 347.1615$; found, 347.1619 .

6-(1-methyl-1H-pyrazol-4-yl)-N-(3-nitrobenzyl)pyrido[2,3-d]pyrimidin-4-amine (7d)

Compound 7d was synthesized from $\mathbf{5 d}$ and $\mathbf{2 0}$ following the similar procedure 
described above for the preparation of 7a (yield, 74\%). mp 290-293 ${ }^{\circ} \mathrm{C} .{ }^{1} \mathrm{H}$ NMR (400 MHz, DMSO-d $d_{6} \delta 9.41(\mathrm{~s}, 1 \mathrm{H}), 9.28(\mathrm{~d}, J=1.6 \mathrm{~Hz}, 1 \mathrm{H}), 9.05(\mathrm{~s}, 1 \mathrm{H}), 8.54(\mathrm{~s}, 1 \mathrm{H})$, 8.35 (s, 1H), $8.27(\mathrm{~s}, 1 \mathrm{H}), 8.13(\mathrm{~d}, J=8.0 \mathrm{~Hz}, 1 \mathrm{H}), 8.06(\mathrm{~s}, 1 \mathrm{H}), 7.88$ (d, $J=7.2 \mathrm{~Hz}$, $1 \mathrm{H}), 7.64(\mathrm{t}, J=7.8 \mathrm{~Hz}, 1 \mathrm{H}), 4.94(\mathrm{~s}, 2 \mathrm{H}), 3.93(\mathrm{~s}, 3 \mathrm{H}) .{ }^{13} \mathrm{C}$ NMR $(101 \mathrm{MHz}$, DMSO- $\left.d_{6}\right) \delta 161.0,157.8,157.0,153.6,148.3,142.1,136.8,134.6,130.3,128.8$, 127.0, 126.7, 122.4, 122.3, 118.7, 110.2, 66.8, 43.8. ESI-MS m/z: $362.2[\mathrm{M}+\mathrm{H}]^{+}$. HRMS, ESI ${ }^{+}, \mathrm{m} / \mathrm{z}$ : Calcd for $\mathrm{C}_{18} \mathrm{H}_{15} \mathrm{~N}_{7} \mathrm{O}_{2}(\mathrm{M}+\mathrm{H})^{+}, 362.1360$; found, 362.1363 .

\section{6-bromoquinazolin-4-ol (9)}

Compound 9 was synthesized from 8 and formamide following the similar procedure described above for the preparation of $\mathbf{3}$, and the crude product was directly used in the next step.

\section{6-bromo-4-chloroquinazoline (10)}

Compound 10 was synthesized from 9 and thionyl chloride and DMF following the similar procedure described above for the preparation of 4 (yield, 75\%). ${ }^{1} \mathrm{H}$ NMR $\left(400 \mathrm{MHz}, \mathrm{DMSO}-d_{6}\right) \delta 8.36(\mathrm{~s}, 1 \mathrm{H}), 8.22(\mathrm{~d}, J=2.4 \mathrm{~Hz}, 1 \mathrm{H}), 8.01(\mathrm{dd}, J=2.4,8.8$ $\mathrm{Hz}, 1 \mathrm{H}), 7.68(\mathrm{~d}, J=8.4 \mathrm{~Hz}, 1 \mathrm{H})$, ESI-MS m/z: $244.9[\mathrm{M}+\mathrm{H}]^{+}$.

6-bromo-N-(3-methoxybenzyl)quinazolin-4-amine (11a)

To a solution of compound $\mathbf{1 0}(2.43 \mathrm{~g}, 0.01 \mathrm{~mol})$ in $25 \mathrm{~mL}$ dioxane was added (3-methoxyphenyl)methanamine (2.06 g, $0.015 \mathrm{~mol})$ and $\mathrm{K}_{2} \mathrm{CO}_{3}(2.76 \mathrm{~g}, 0.02 \mathrm{~mol})$, the reaction mixture was stirred at room temperature overnight. After that, the mixture was poured into $150 \mathrm{~mL}$, and then the resulting precipitate was filtered, washed with $50 \%$ ethanol, and dried overnight under vacuum to give 11a as white solid (2.33 g, yield, 68\%). ${ }^{1} \mathrm{H}$ NMR (400 MHz, DMSO- $\left.d_{6}\right) \delta 8.90(\mathrm{t}, J=5.4 \mathrm{~Hz}, 1 \mathrm{H}), 8.63(\mathrm{~s}, 1 \mathrm{H})$, $8.50(\mathrm{~s}, 1 \mathrm{H}), 7.90(\mathrm{~d}, J=8.8 \mathrm{~Hz}, 1 \mathrm{H}), 7.65(\mathrm{~d}, J=9.2 \mathrm{~Hz}, 1 \mathrm{H}), 7.24(\mathrm{t}, J=8.0 \mathrm{~Hz}$, $1 \mathrm{H}), 6.94(\mathrm{~d}, J=6.4 \mathrm{~Hz}, 2 \mathrm{H}), 6.82(\mathrm{~d}, J=7.6 \mathrm{~Hz}, 1 \mathrm{H}), 4.75(\mathrm{~d}, J=5.6 \mathrm{~Hz}, 2 \mathrm{H}), 3.73$ (s, 3H). ESI-MS m/z: $344.4[\mathrm{M}+\mathrm{H}]^{+}$.

\section{6-bromo-N-(1-(2,6-dichloro-3-fluorophenyl)ethyl)quinazolin-4-amine (11b)}

Compound 11b was synthesized from 10 and 1-(2,6-Dichloro-3-fluorophenyl)ethan-1-amine following the similar procedure described above for the preparation of $11 \mathrm{a}$ (yield, 71\%). ${ }^{1} \mathrm{H}$ NMR (400 MHz, DMSO- $\left.d_{6}\right) \delta 8.91(\mathrm{~d}, J=2.0 \mathrm{~Hz}, 1 \mathrm{H}), 8.76(\mathrm{~d}$, $J=5.6 \mathrm{~Hz}, 1 \mathrm{H}), 8.33(\mathrm{~s}, 1 \mathrm{H}), 7.90(\mathrm{dd}, J=2.0,8.8 \mathrm{~Hz}, 1 \mathrm{H}), 7.61(\mathrm{~d}, J=9.2 \mathrm{~Hz}, 1 \mathrm{H})$, $7.45-7.42(\mathrm{~m}, 1 \mathrm{H}), 7.32(\mathrm{t}, J=8.8 \mathrm{~Hz}, 1 \mathrm{H}), 5.85(\mathrm{q}, J=7.2 \mathrm{~Hz}, 1 \mathrm{H}), 1.70(\mathrm{~d}, J=7.2$ 
Hz, 3H). ESI-MS m/z: $416.4[\mathrm{M}+\mathrm{H}]^{+}$.

6-bromo-4-(1-(2,6-dichloro-3-fluorophenyl)ethoxy)quinazoline (11c)

To a solution of 1-(2,6-Dichloro-3-fluorophenyl)ethan-1-ol (2.71 g, $0.013 \mathrm{~mol})$ in $15 \mathrm{~mL}$ dioxane. The resulting solution was cooled at $0{ }^{\circ} \mathrm{C}$ and slowly added $\mathrm{NaH}$ (2.00 g, $0.03 \mathrm{~mol}, 60 \%$ dispersion in mineral oil) over $15 \mathrm{~min}$. After complete addition, compound $\mathbf{1 0}$ was added. The mixture was stirred at $0{ }^{\circ} \mathrm{C}$ for $1 \mathrm{~h}$ and then stirred at room temperature overnight. The reaction mixture was poured into $100 \mathrm{~mL}$ $\mathrm{H}_{2} \mathrm{O}$, and the $\mathrm{pH}$ was adjusted to neutral with $10 \%$ aqueous hydrochloric acid. The resulting precipitate was filtered and washed with $50 \%$ ethanol, and dried overnight under high vacuum to give 11c as white solid (3.30 g, yield, 79\%). ${ }^{1} \mathrm{H}$ NMR (400 MHz, DMSO- $\left.d_{6}\right) \delta 8.73(\mathrm{~s}, 1 \mathrm{H}), 8.36(\mathrm{~d}, J=2.0 \mathrm{~Hz}, 1 \mathrm{H}), 8.10(\mathrm{dd}, J=2.0,8.8 \mathrm{~Hz}$, $1 \mathrm{H}), 7.86(\mathrm{~d}, J=9.2 \mathrm{~Hz}, 1 \mathrm{H}), 7.54(\mathrm{dd}, J=5.0,9.2 \mathrm{~Hz}, 1 \mathrm{H}), 7.43(\mathrm{t}, J=8.8 \mathrm{~Hz}, 1 \mathrm{H})$, $6.90(\mathrm{q}, J=6.8 \mathrm{~Hz}, 1 \mathrm{H}), 1.86(\mathrm{~d}, J=6.8 \mathrm{~Hz}, 3 \mathrm{H})$. ESI-MS m/z: $417.6[\mathrm{M}+\mathrm{H}]^{+}$.

6-bromo-N-(3-chloro-4-fluorophenyl)quinazolin-4-amine (11d)

To a solution of compound $\mathbf{1 0}(2.43 \mathrm{~g}, 0.01 \mathrm{~mol})$ and 3-chloro-4-fluoroaniline $1.45 \mathrm{~g}(0.01 \mathrm{~mol})$ in acetone : $\mathrm{H}_{2} \mathrm{O}(4: 1)(25 \mathrm{~mL})$, followed by a few drops of hydrochloric acid. The reaction mixture stirred at reflux for $3 \mathrm{~h}$, after cooling to room temperature, the $\mathrm{pH}$ was adjusted to neutral using ammonium hydroxide. The resulting precipitate was filtered and washed with water and dried overnight under high vacuum to give 11d as a earthy yellow solid (2.80 g, yield, 80\%). ${ }^{1} \mathrm{H}$ NMR (400 MHz, DMSO- $\left.d_{6}\right) \delta 11.73(\mathrm{~s}, 1 \mathrm{H}), 9.28(\mathrm{~d}, J=2.0 \mathrm{~Hz}, 1 \mathrm{H}), 8.97(\mathrm{~s}, 1 \mathrm{H}), 8.23$ (dd, $J=$ $2.0,8.8 \mathrm{~Hz}, 1 \mathrm{H}), 8.10(\mathrm{dd}, J=2.4,6.8 \mathrm{~Hz}, 1 \mathrm{H}), 7.94(\mathrm{~d}, J=9.2 \mathrm{~Hz}, 1 \mathrm{H}), 7.80$ (ddd, $J$ $=2.8,4.4,7.2 \mathrm{~Hz}, 1 \mathrm{H}), 7.55(\mathrm{t}, J=9.0 \mathrm{~Hz}, 1 \mathrm{H}) . \mathrm{ESI}-\mathrm{MS} \mathrm{m} / \mathrm{z}: 354.3[\mathrm{M}+\mathrm{H}]^{+}$.

\section{6-bromo-N-(quinolin-6-yl)quinazolin-4-amine (11e)}

Compound 11e was synthesized from 10 and 6-quinolylamine following the similar procedure described above for preparation of 11d (yield, 74\%). ${ }^{1} \mathrm{H}$ NMR (400 MHz, DMSO- $\left.d_{6}\right) \delta 12.19(\mathrm{~s}, 1 \mathrm{H}), 9.45(\mathrm{~d}, J=1.6 \mathrm{~Hz}, 1 \mathrm{H}), 9.19(\mathrm{dd}, J=1.2,4.8 \mathrm{~Hz}$, $1 \mathrm{H}), 9.03(\mathrm{~s}, 1 \mathrm{H}), 8.99$ (d, $J=8.0 \mathrm{~Hz}, 1 \mathrm{H}), 8.71(\mathrm{~d}, J=2.0 \mathrm{~Hz}, 1 \mathrm{H}), 8.47$ (dd, $J=2.0$, $9.2 \mathrm{~Hz}, 1 \mathrm{H}), 8.41(\mathrm{~d}, J=9.2 \mathrm{~Hz}, 1 \mathrm{H}), 8.27(\mathrm{dd}, J=2.0,8.8 \mathrm{~Hz}, 1 \mathrm{H}), 8.01(\mathrm{~d}, J=8.8$ $\mathrm{Hz}, 1 \mathrm{H}), 7.96(\mathrm{dd}, J=5.2,8.4 \mathrm{~Hz}, 1 \mathrm{H}) . \mathrm{ESI}-\mathrm{MS} \mathrm{m} / \mathrm{z}: 353.7[\mathrm{M}+\mathrm{H}]^{+}$.

\section{6-bromo-N-(3-ethynylphenyl)quinazolin-4-amine (11f)}

Compound 11 was synthesized from 10 and 3-ethynylaniline following the same 
procedure described above for preparation of 11d (yield, 86\%). ${ }^{1} \mathrm{H}$ NMR (400 MHz, DMSO-d $\left.d_{6}\right) \delta 11.83(\mathrm{~s}, 1 \mathrm{H}), 9.34(\mathrm{~d}, J=1.6 \mathrm{~Hz}, 1 \mathrm{H}), 8.99(\mathrm{~s}, 1 \mathrm{H}), 8.25(\mathrm{dd}, J=2.0$, $8.8 \mathrm{~Hz}, 1 \mathrm{H}), 7.97$ (d, $J=8.8 \mathrm{~Hz}, 1 \mathrm{H}), 7.94(\mathrm{t}, J=1.6 \mathrm{~Hz}, 1 \mathrm{H}), 7.84-7.81(\mathrm{~m}, 1 \mathrm{H})$, $7.51(\mathrm{t}, J=8.0 \mathrm{~Hz}, 1 \mathrm{H}), 7.44-7.42(\mathrm{~m}, 1 \mathrm{H}), 4.30(\mathrm{~s}, 1 \mathrm{H})$. ESI-MS m/z: 326.2 $[\mathrm{M}+\mathrm{H}]^{+}$.

\section{6-bromo-4-(quinolin-6-yloxy)quinazoline (11g)}

To a solution of 6-hydroxyquinoline $(1.60 \mathrm{~g}, 0.011 \mathrm{~mol})$ in $20 \mathrm{~mL}$ dioxane was added cesium carbonate $(7.20 \mathrm{~g}, 0.022 \mathrm{~mol})$. After stirring at room temperature for $1 \mathrm{~h}$, compound $\mathbf{1 0}$ was added. The mixture was stirred at room temperature for a further overnight. The reaction mixture was poured into $200 \mathrm{~mL} \mathrm{H}_{2} \mathrm{O}$, and $\mathrm{pH}$ was adjusted to neutral using aqueous hydrochloric acid. The resulting precipitate was filtered and washed with water and dried overnight under high vacuum to give $\mathbf{1 1 g}$ as white solid (2.10 g, yield, 60\%). ${ }^{1} \mathrm{H}$ NMR (400 MHz, DMSO- $\left.d_{6}\right) \delta 8.96$ (d, $J=2.8 \mathrm{~Hz}$, $1 \mathrm{H}), 8.79(\mathrm{~s}, 1 \mathrm{H}), 8.62(\mathrm{~d}, J=2.0 \mathrm{~Hz}, 1 \mathrm{H}), 8.41(\mathrm{~d}, J=8.0 \mathrm{~Hz}, 1 \mathrm{H}), 8.22(\mathrm{dd}, J=2.0$, $8.8 \mathrm{~Hz}, 1 \mathrm{H}), 8.16(\mathrm{~d}, J=9.2 \mathrm{~Hz}, 1 \mathrm{H}), 8.00(\mathrm{~d}, J=9.2 \mathrm{~Hz}, 2 \mathrm{H}), 7.82(\mathrm{dd}, J=2.8,9.2$ $\mathrm{Hz}, 1 \mathrm{H}), 7.61(\mathrm{dd}, J=4.0,8.4 \mathrm{~Hz}, 1 \mathrm{H})$. ESI-MS m/z: $354.8[\mathrm{M}+\mathrm{H}]^{+}$.

N-(3-methoxybenzyl)-6-(1-methyl-1H-pyrazol-4-yl)quinazolin-4-amine (12a)

Compound 12a was synthesized from 11a and 20 following the similar procedure described above for the preparation of $7 \mathbf{a}$ (yield, 47\%). mp 167-169 ${ }^{\circ} \mathrm{C} .{ }^{1} \mathrm{H}$ NMR (400 MHz, DMSO- $\left.d_{6}\right) \delta 8.71(\mathrm{~s}, 1 \mathrm{H}), 8.53(\mathrm{~s}, 1 \mathrm{H}), 8.41(\mathrm{~s}, 1 \mathrm{H}), 8.20(\mathrm{~s}, 1 \mathrm{H})$, $7.99(\mathrm{~d}, J=8.8 \mathrm{~Hz}, 2 \mathrm{H}), 7.68(\mathrm{~d}, J=8.8 \mathrm{~Hz}, 1 \mathrm{H}), 7.25(\mathrm{t}, J=7.6 \mathrm{~Hz}, 1 \mathrm{H}), 6.97(\mathrm{~s}$, $2 \mathrm{H}), 6.83(\mathrm{~d}, J=8.4 \mathrm{~Hz}, 1 \mathrm{H}), 4.81(\mathrm{~d}, J=3.6 \mathrm{~Hz}, 2 \mathrm{H}), 3.91(\mathrm{~s}, 3 \mathrm{H}), 3.73(\mathrm{~s}, 3 \mathrm{H}) .{ }^{13} \mathrm{C}$ NMR (101 MHz, DMSO- $\left.d_{6}\right) \delta 159.8,159.6,154.8,148.0,141.5,136.7,130.6,130.5$, $129.9,128.5,128.4,121.9,119.8,117.9,115.6,113.6,112.5,55.4,43.9,39.2$. ESI-MS m/z: $346.2[\mathrm{M}+\mathrm{H}]^{+}$. HRMS, ESI ${ }^{+}, \mathrm{m} / \mathrm{z}$ : Calcd for $\mathrm{C}_{20} \mathrm{H}_{19} \mathrm{~N}_{5} \mathrm{O}(\mathrm{M}+\mathrm{H})^{+}, 346.1662$; found, 346.1666 .

4-(1-(2,6-dichloro-3-fluorophenyl)ethoxy)-6-(1-methyl-1H-pyrazol-4-yl)quinazoline $(12 b)$

Compound 12b was synthesized from 11c and 20 following the similar procedure described above for the preparation of $7 \mathbf{a}$ (yield, 47\%). mp 163-165 ${ }^{\circ} \mathrm{C} .{ }^{1} \mathrm{H}$ NMR (400 MHz, DMSO- $\left.d_{6}\right) \delta 8.62(\mathrm{~s}, 1 \mathrm{H}), 8.36(\mathrm{~s}, 1 \mathrm{H}), 8.30(\mathrm{~d}, J=2.0 \mathrm{~Hz}, 1 \mathrm{H})$, $8.18(\mathrm{dd}, J=2.0,8.8 \mathrm{~Hz}, 1 \mathrm{H}), 8.02(\mathrm{~s}, 1 \mathrm{H}), 7.88(\mathrm{~d}, J=8.8 \mathrm{~Hz}, 1 \mathrm{H}), 7.53(\mathrm{dd}, J=4.8$, 
$8.8 \mathrm{~Hz}, 1 \mathrm{H}), 7.42(\mathrm{t}, J=8.8 \mathrm{~Hz}, 1 \mathrm{H}), 6.94(\mathrm{q}, J=6.8 \mathrm{~Hz}, 1 \mathrm{H}), 3.93(\mathrm{~s}, 3 \mathrm{H}), 1.88(\mathrm{~d}, J$ $=7.2 \mathrm{~Hz}, 3 \mathrm{H}) .{ }^{13} \mathrm{C}$ NMR $\left(101 \mathrm{MHz}, \mathrm{DMSO}-d_{6}\right) \delta 164.9,153.4,149.6,137.6,136.9$, 132.6, 129.1, 128.5, 121.2, 117.7, 117.5, 117.3, 116.6, 71.5, 39.2, 25.4, 18.5. ESI-MS m/z: $417.3[\mathrm{M}+\mathrm{H}]^{+}$. HRMS, ESI ${ }^{+}, \mathrm{m} / \mathrm{z}$ : Calcd for $\mathrm{C}_{20} \mathrm{H}_{15} \mathrm{Cl}_{2} \mathrm{FN}_{4} \mathrm{O}(\mathrm{M}+\mathrm{H})^{+}, 417.0680$; found, 417.0685 .

N-(3-chloro-4-fluorophenyl)-6-(1-methyl-1H-pyrazol-4-yl)quinazolin-4-amine (12c)

Compound 12c was synthesized from 11d and $\mathbf{2 0}$ following the similar procedure described above for the preparation of $7 \mathbf{a}$ (yield, 69\%). mp 209-211 ${ }^{\circ} \mathrm{C} .{ }^{1} \mathrm{H}$ NMR (400 MHz, DMSO- $\left.d_{6}\right) \delta 9.78(\mathrm{~s}, 1 \mathrm{H}), 8.61(\mathrm{~d}, J=21.6 \mathrm{~Hz}, 2 \mathrm{H}), 8.22(\mathrm{~d}, J=$ $23.6 \mathrm{~Hz}, 2 \mathrm{H}), 8.05(\mathrm{~s}, 2 \mathrm{H}), 7.82(\mathrm{~d}, J=27.6 \mathrm{~Hz}, 2 \mathrm{H}), 7.46(\mathrm{~s}, 1 \mathrm{H}), 3.93(\mathrm{~s}, 3 \mathrm{H}) .{ }^{13} \mathrm{C}$ NMR (101 MHz, DMSO- $\left.d_{6}\right) \delta 157.6,155.0,153.9,152.6,148.6,136.9,131.2,128.8$, 124.1, 122.9, 121.8, 119.4, 119.2, 117.7, 117.1, 116.9, 115.8, 39.9. ESI-MS m/z: 354.6 $[\mathrm{M}+\mathrm{H}]^{+}$. HRMS, ESI ${ }^{+}, \mathrm{m} / \mathrm{z}$ : Calcd for $\mathrm{C}_{21} \mathrm{H}_{16} \mathrm{~N}_{6}(\mathrm{M}+\mathrm{H})^{+}$, 354.0916; found, 354.0919. 6-(1-methyl-1H-pyrazol-4-yl)-N-(quinolin-6-yl)quinazolin-4-amine (12d)

Compound 12d was synthesized from 11e and 20 following the similar procedure described above for the preparation of $7 \mathbf{a}$ (yield, 79\%). mp 263-265 ${ }^{\circ} \mathrm{C} .{ }^{1} \mathrm{H}$ NMR (400 MHz, DMSO-d 6 ) $\delta 10.02(\mathrm{~s}, 1 \mathrm{H}), 8.84(\mathrm{dd}, J=1.6,4.0 \mathrm{~Hz}, 1 \mathrm{H}), 8.78(\mathrm{~d}, J$ $=1.6 \mathrm{~Hz}, 1 \mathrm{H}), 8.65(\mathrm{~s}, 1 \mathrm{H}), 8.57(\mathrm{~d}, J=2.0 \mathrm{~Hz}, 1 \mathrm{H}), 8.37$ (d, $J=7.6 \mathrm{~Hz}, 1 \mathrm{H}), 8.31(\mathrm{~s}$, $1 \mathrm{H}), 8.24(\mathrm{dd}, J=2.0,8.8 \mathrm{~Hz}, 1 \mathrm{H}), 8.17-8.07$ (m, 3H), 7.82 (d, $J=8.8 \mathrm{~Hz}, 1 \mathrm{H}), 7.53$ $(\mathrm{dd}, J=4.0,8.0 \mathrm{~Hz}, 1 \mathrm{H}), 3.95$ (s, 3H). ${ }^{13} \mathrm{C}$ NMR (101MHz, DMSO-d 6$) \delta 157.8,154.1$, $149.7,148.7,145.4,137.7,136.9,135.9,131.4,131.2$, 129.5, 128.8, 128.6, 126.6, 122.2, 121.8, 118.6, 117.9, 116.0, 99.9, 39.2. ESI-MS m/z: $353.6[\mathrm{M}+\mathrm{H}]^{+}$. HRMS, $\mathrm{ESI}^{+}, \mathrm{m} / \mathrm{z}$ : Calcd for $\mathrm{C}_{21} \mathrm{H}_{16} \mathrm{~N}_{6}(\mathrm{M}+\mathrm{H})^{+}, 353.1509$; found, 353.1512 .

N-(3-ethynylphenyl)-6-(1-methyl-1H-pyrazol-4-yl)quinazolin-4-amine (12e)

Compound 12e was synthesized from $11 f$ and 20 following the similar procedure described above for the preparation of 7a. The crude product was purified by flash chromatography on $\mathrm{C}_{18}$ reverse phase silica gel eluting with $35 \%$ methnaol/water ( $50 \mathrm{mmol}$ ammonium acetate adjusted to $\mathrm{pH}=4$ with formic acid) to give compound 12e (yield, 37\%). mp 118-121 ${ }^{\circ} \mathrm{C} .{ }^{1} \mathrm{H}$ NMR (400 MHz, DMSO-d 6 ) $\delta 9.79$ (s, 1H), $8.70(\mathrm{~s}, 1 \mathrm{H}), 8.60(\mathrm{~s}, 1 \mathrm{H}), 8.28(\mathrm{~s}, 1 \mathrm{H}), 8.10-8.06(\mathrm{~m}, 3 \mathrm{H}), 7.96(\mathrm{~d}, J=8.0 \mathrm{~Hz}, 1 \mathrm{H})$, $7.79(\mathrm{~d}, J=8.4 \mathrm{~Hz}, 1 \mathrm{H}), 7.45(\mathrm{t}, J=7.8 \mathrm{~Hz}, 1 \mathrm{H}), 7.27(\mathrm{~d}, J=7.6 \mathrm{~Hz}, 1 \mathrm{H}), 4.23$ (s, 
1H), $3.93(\mathrm{~s}, 3 \mathrm{H}) .{ }^{13} \mathrm{C}$ NMR (101 MHz, DMSO- $\left.d_{6}\right) \delta 157.7,153.9,148.3,139.8$, 136.9, 131.4, 131.3, 129.4, 128.8, 128.6, 127.3, 125.5, 123.3, 122.3, 121.8, 117.8, 115.9, 83.8, 81.1, 39.2. ESI-MS m/z: $326.6[\mathrm{M}+\mathrm{H}]^{+}$. HRMS, ESI ${ }^{+}, \mathrm{m} / \mathrm{z}:$ Calcd for $\mathrm{C}_{21} \mathrm{H}_{16} \mathrm{~N}_{6}(\mathrm{M}+\mathrm{H})^{+}, 326.1400$; found, 326.1406.

6-(1-methyl-1H-pyrazol-4-yl)-4-(quinolin-6-yloxy)quinazoline (12f)

Compund $12 \mathrm{f}$ was synthesized from $\mathbf{1 1 g}$ and $\mathbf{2 0}$ following the same procedure described above for preparation of $7 \mathbf{a}$ (yield, 83\%). mp 209-211 ${ }^{\circ} \mathrm{C} .{ }^{1} \mathrm{H}$ NMR (400 MHz, DMSO- $\left.d_{6}\right) \delta 8.96(\mathrm{dd}, J=1.6,4.0 \mathrm{~Hz}, 1 \mathrm{H}), 8.67(\mathrm{~s}, 1 \mathrm{H}), 8.55(\mathrm{~d}, J=2.0 \mathrm{~Hz}$, $1 \mathrm{H}), 8.49(\mathrm{~s}, 1 \mathrm{H}), 8.42(\mathrm{dd}, J=1.2,6.8 \mathrm{~Hz}, 1 \mathrm{H}), 8.31(\mathrm{dd}, J=2.0,8.8 \mathrm{~Hz}, 1 \mathrm{H}), 8.16$ (t, $J=4.6 \mathrm{~Hz}, 2 \mathrm{H}), 8.02(\mathrm{dd}, J=4.4,7.2 \mathrm{~Hz}, 2 \mathrm{H}), 7.83(\mathrm{dd}, J=2.4,8.8 \mathrm{~Hz}, 1 \mathrm{H}), 7.61$ $(\mathrm{dd}, J=4.0,8.4 \mathrm{~Hz}, 1 \mathrm{H}), 3.92(\mathrm{~s}, 3 \mathrm{H}) .{ }^{13} \mathrm{C}$ NMR $\left(101 \mathrm{MHz}, \mathrm{DMSO}-d_{6}\right) \delta 166.6$, $153.3,150.9,150.4,150.2,146.2,137.1,136.1,133.1,132.9,131.0,129.4,128.9$, 128.6, 126.1, 122.4, 121.1, 119.4, 117.6, 116.6, 39.2. MS: m/z (ESI+) $354.5[\mathrm{M}+\mathrm{H}]^{+}$. HRMS, ESI ${ }^{+}, \mathrm{m} / \mathrm{z}$ : Calcd for $\mathrm{C}_{21} \mathrm{H}_{15} \mathrm{~N}_{5} \mathrm{O}(\mathrm{M}+\mathrm{H})^{+}, 354.1349$; found, 354.1348.

N-(1-(2,6-dichloro-3-fluorophenyl)ethyl)-6-(1-(piperidin-4-yl)-1H-pyrazol-4-yl)quina zolin-4-amine (13a)

Compound 13a was synthesized from 11b and 21 following the similar procedure described above for the preparation of $\mathbf{6}$ (yield, $79 \%$ ). mp $185-188^{\circ} \mathrm{C} .{ }^{1} \mathrm{H}$ NMR (400 MHz, MeOD) $\delta 8.65$ (d, $J=1.6 \mathrm{~Hz}, 1 \mathrm{H}), 8.29$ (s, 2H), 8.15 (s, 1H), 8.03 $(\mathrm{dd}, J=2.0,8.4 \mathrm{~Hz}, 1 \mathrm{H}), 7.70(\mathrm{~d}, J=8.8 \mathrm{~Hz}, 1 \mathrm{H}), 7.35(\mathrm{dd}, J=4.8,8.8 \mathrm{~Hz}, 1 \mathrm{H}), 7.13$ $(\mathrm{t}, J=8.6 \mathrm{~Hz}, 1 \mathrm{H}), 6.10(\mathrm{q}, J=7.2 \mathrm{~Hz}, 1 \mathrm{H}), 4.64(\mathrm{ddd}, J=4.4,12.6,17.0 \mathrm{~Hz}, 1 \mathrm{H})$, $3.61(\mathrm{dt}, J=3.2,16.4 \mathrm{~Hz}, 2 \mathrm{H}), 3.27(\mathrm{td}, J=3.6,12.8 \mathrm{~Hz}, 2 \mathrm{H}), 2.45-2.30(\mathrm{~m}, 4 \mathrm{H})$, $1.84(\mathrm{~d}, J=7.2 \mathrm{~Hz}, 3 \mathrm{H}) .{ }^{13} \mathrm{C}$ NMR (101 MHz, MeOD) $\delta 158.6,158.4,155.9,154.0$, $146.8,140.0,136.9,130.8,130.5,129.4,129.3,126.7,126.0,122.1,117.6,115.0$, 114.8, 55.7, 49.1, 42.8, 28.9, 16.4. ESI-MS m/z: $485.5[\mathrm{M}+\mathrm{H}]^{+} . \mathrm{HRMS}, \mathrm{ESI}^{+}, \mathrm{m} / \mathrm{z}$ : Calcd for $\mathrm{C}_{24} \mathrm{H}_{23} \mathrm{Cl}_{2} \mathrm{FN}_{6}(\mathrm{M}+\mathrm{H})^{+}$, 485.1418; found, 485.1420.

4-(1-(2,6-dichloro-3-fluorophenyl)ethoxy)-6-(1-(piperidin-4-yl)-1H-pyrazol-4-yl)quin azoline (13b)

Compound 13b was synthesized from 11c and 21 following the similar procedure described above for the preparation of $\mathbf{6}$ (yield, $50 \%$ ). mp $157-158^{\circ} \mathrm{C} .{ }^{1} \mathrm{H}$ NMR (400 MHz, MeOD) $\delta 8.56$ (s, 1H), 8.39 (s, 1H), 8.26 (s, 1H), 8.14 (dd, $J=1.2$, $8.4 \mathrm{~Hz} 1 \mathrm{H}), 7.99$ (s, 1H), $7.85(\mathrm{~d}, J=8.8 \mathrm{~Hz}, 1 \mathrm{H}), 7.41(\mathrm{dd}, J=4.8,8.8 \mathrm{~Hz}, 1 \mathrm{H}), 7.21$ 
(t, $J=8.6 \mathrm{~Hz}, 1 \mathrm{H}), 7.02(\mathrm{q}, J=6.8 \mathrm{~Hz}, 1 \mathrm{H}), 4.49-4.42(\mathrm{~m}, 1 \mathrm{H}), 3.36(\mathrm{~d}, J=7.2 \mathrm{~Hz}$, $1 \mathrm{H}), 3.29(\mathrm{~s}, 1 \mathrm{H}), 2.96-2.90(\mathrm{~m}, 2 \mathrm{H}), 2.25-2.01(\mathrm{~m}, 5 \mathrm{H}), 1.94(\mathrm{~d}, J=6.8 \mathrm{~Hz}, 3 \mathrm{H})$. ${ }^{13} \mathrm{C}$ NMR (101 MHz, MeOD) $\delta 165.3,158.5,156.0,153.1,148.9,138.5,137.5,136.3$, 132.1, 129.8, 128.7, 127.0, 125.7, 121.3, 118.0, 116.5, 104.9, 72.0, 58.4, 44.1, 31.7, 17.0. ESI-MS m/z: $486.6[\mathrm{M}+\mathrm{H}]^{+}$. HRMS, ESI ${ }^{+}, \mathrm{m} / \mathrm{z}$ : Calcd for $\mathrm{C}_{24} \mathrm{H}_{22} \mathrm{Cl}_{2} \mathrm{FN}_{5} \mathrm{O}$ $(\mathrm{M}+\mathrm{H})^{+}, 486.1258$; found, 486.1254 .

N-(3-chloro-4-fluorophenyl)-6-(1-(piperidin-4-yl)-1H-pyrazol-4-yl)quinazolin-4-amin $-e(13 \mathrm{c})$

Compound 13c was synthesized from 11d and 21 following the similar procedure described above for the preparation of 6 (yield, 63\%). mp 293-296 ${ }^{\circ} \mathrm{C} .{ }^{1} \mathrm{H}$ NMR (400 MHz, DMSO-d $\left.)_{6}\right) 9.82(\mathrm{~s}, 1 \mathrm{H}), 8.67$ (d, $\left.J=1.4 \mathrm{~Hz}, 1 \mathrm{H}\right), 8.59(\mathrm{~s}, 1 \mathrm{H})$, $8.36(\mathrm{~s}, 1 \mathrm{H}), 8.20(\mathrm{dd}, J=2.4,6.8 \mathrm{~Hz}, 1 \mathrm{H}), 8.12$ (dd, $J=2.0,8.8 \mathrm{~Hz}, 1 \mathrm{H}), 8.08$ (s, 1H), 7.87 (ddd, $J=2.4,5.6,7.6 \mathrm{~Hz}, 1 \mathrm{H}), 7.79(\mathrm{~d}, J=8.8 \mathrm{~Hz}, 1 \mathrm{H}), 7.47(\mathrm{t}, J=9.2 \mathrm{~Hz}$, $1 \mathrm{H}), 4.29-4.22(\mathrm{~m}, 1 \mathrm{H}), 3.10-2.99(\mathrm{~m}, 3 \mathrm{H}), 2.66-2.55(\mathrm{~m}, 2 \mathrm{H}), 2.03(\mathrm{~d}, J=10.0$ $\mathrm{Hz}, 2 \mathrm{H}), 1.89-1.82(\mathrm{~m}, 2 \mathrm{H}) .{ }^{13} \mathrm{C}$ NMR (101 MHz, DMSO- $\left.d_{6}\right) \delta$ 157.6, 155.0 153.9, 152.6, 148.6, 136.5, 131.6, 128.7, 125.8, 124.1, 123.0, 121.2, 119.4, 117.7, 117.1, 116.9, 115.8, 59.8, 45.4, 33.9, 33.7, 22.9. ESI-MS m/z: $423.5[\mathrm{M}+\mathrm{H}]^{+}$. HRMS, ESI ${ }^{+}$, $\mathrm{m} / \mathrm{z}$ : Calcd for $\mathrm{C}_{24} \mathrm{H}_{22} \mathrm{Cl}_{2} \mathrm{FN}_{5} \mathrm{O}(\mathrm{M}+\mathrm{H})^{+}$, 423.1495; found, 423.1499.

6-(1-(piperidin-4-yl)-1H-pyrazol-4-yl)-N-(quinolin-6-yl)quinazolin-4-amine (13d)

Compound 13d was synthesized from 11e and 21 following the similar procedure described above for the preparation of 6 (yield, 63\%). mp 126-127 ${ }^{\circ} \mathrm{C} .{ }^{1} \mathrm{H}$ NMR (400 MHz, DMSO- $\left.d_{6}\right) \delta 10.02(\mathrm{~s}, 1 \mathrm{H}), 8.84(\mathrm{dd}, J=1.6,4.4 \mathrm{~Hz}, 1 \mathrm{H}), 8.78(\mathrm{~d}, J$ $=1.2 \mathrm{~Hz}, 1 \mathrm{H}), 8.64(\mathrm{~s}, 1 \mathrm{H}), 8.57(\mathrm{~d}, J=2.0 \mathrm{~Hz}, 1 \mathrm{H}), 8.39-8.35(\mathrm{~m}, 2 \mathrm{H}), 8.25(\mathrm{dd}, J$ $=2.4,9.2 \mathrm{~Hz}, 1 \mathrm{H}), 8.16-8.12(\mathrm{~m}, 2 \mathrm{H}), 8.07(\mathrm{~d}, J=9.2 \mathrm{~Hz}, 1 \mathrm{H}), 7.81(\mathrm{~d}, J=8.4 \mathrm{~Hz}$, $1 \mathrm{H}), 7.52(\mathrm{dd}, J=4.0,8.0 \mathrm{~Hz}, 1 \mathrm{H}), 4.29-4.24(\mathrm{~m}, 1 \mathrm{H}), 3.09$ (d, $J=12.0 \mathrm{~Hz}, 2 \mathrm{H})$, $2.63(\mathrm{t}, J=11.8 \mathrm{~Hz}, 2 \mathrm{H}), 2.06-2.03(\mathrm{~m}, 2 \mathrm{H}), 1.90-1.81(\mathrm{~m}, 2 \mathrm{H}) .{ }^{13} \mathrm{C} \mathrm{NMR}(101$ MHz, DMSO- $\left.d_{6}\right) \delta 157.8,154.0,149.6,148.7,145.4,137.7,136.6,135.9,131.6$, 131.3, 129.4, 128.8, 128.6, 126.6, 125.8, 122.2, 121.3, 118.6, 117.9, 116.0, 59.8, 45.4, 33.9. ESI-MS m/z: $422.7[\mathrm{M}+\mathrm{H}]^{+}$. HRMS, ESI ${ }^{+}, \mathrm{m} / \mathrm{z}$ : Calcd for $\mathrm{C}_{25} \mathrm{H}_{23} \mathrm{~N}_{7}(\mathrm{M}+\mathrm{H})^{+}$, 422.2088; found, 422.2093 .

N-(6-Bromo-4-hydroxyquinazolin-2-yl)guanidine (15)

To the mixture of 2-amino-5-bromobenzoic acid (14) $(15.0 \mathrm{~g}, 0.07 \mathrm{~mol})$, water 
$150 \mathrm{~mL}$ and concentrated sulfuric acid $(8 \mathrm{~mL})$ was heated at $100{ }^{\circ} \mathrm{C}$ before being allowed to cool. Dicyandiamide was added, and the reaction mixture was stirred for 2 h at $100{ }^{\circ} \mathrm{C}$. The reaction was cooled and filtered. The white solid was basified with $100 \mathrm{~mL}(4 \mathrm{~mol} / \mathrm{L}) \mathrm{NaOH}$ at $180{ }^{\circ} \mathrm{C}$ for a further $15 \mathrm{~min}$, cooled to room temperature, filtered and washed with water until neutral $\mathrm{pH}$, and dried overnight under vacuum to give $\mathbf{1 5}$ as white solid $11.8 \mathrm{~g}$, which was directly used in the next step.

\section{2-amino-6-bromoquinazolin-4-ol (16)}

To a solution of N-(6-Bromo-4-hydroxyquinazolin-2-yl)guanidine (15) $(8.00 \mathrm{~g}$, $0.028 \mathrm{~mol})$ and 4-Pyridinethiol $(6.20 \mathrm{~g}, 0.056 \mathrm{~mol})$ in ehylene glycol, $\mathrm{KOH}(7.80 \mathrm{~g}$, $0.14 \mathrm{~mol}$ ) was added. The resulting reaction mixture was stirred at $170{ }^{\circ} \mathrm{C}$ for about 4 $\mathrm{h}$, cooled to room temperature, poured into $500 \mathrm{~mL} \mathrm{H}_{2} \mathrm{O}$, and the $\mathrm{pH}$ was adjusted to neutral using hydrochloric acid. The resulting precipitate was filtered, washed with water and 50\% ethanol, and dried overnight under high vacuum to give $\mathbf{1 6}$ as off-white solid (4.60 g, yield, 68\%). ${ }^{1} \mathrm{H}$ NMR (400 MHz, DMSO- $\left.d_{6}\right) \delta 11.11(\mathrm{~s}, 1 \mathrm{H})$, $7.97(\mathrm{~d}, J=2.4 \mathrm{~Hz}, 1 \mathrm{H}), 7.69$ (dd, $J=2.8,8.8 \mathrm{~Hz}, 1 \mathrm{H}), 7.57$ (s, 2H), 7.30 (d, $J=8.8$ $\mathrm{Hz}, 1 \mathrm{H})$. ESI-MS m/z: $242.0[\mathrm{M}+\mathrm{H}]^{+}$.

2-amino-6-bromo-N-(1-(2,6-dichloro-3-fluorophenyl)ethyl)quinazoline-4-amine (17a)

To a solution of compound $16(1.20 \mathrm{~g}, 0.005 \mathrm{~mol})$ and benzotriazol-1-yloxytris(dimethylamino)-phosphonium hexafluorophosphat (BOP) $(2.90 \mathrm{~g}, 0.0065 \mathrm{~mol})$ in 20 $\mathrm{mL}$ acetonitrile, 1,8-Diazabicyclo-[5.4.0]undec-7-ene (DBU) (1.1 mL, $0.0075 \mathrm{~mol})$ was added and the solution became clear. When precipitated solid was formed, 1-(2,6-Dichloro-3-fluoro-phenyl)-ethan-1-amine (1.56 g, $0.0075 \mathrm{~mol})$ was added to the reaction mixture. The reaction mixture was stirred at room temperature overnight, then poured into $100 \mathrm{~mL} \mathrm{H}_{2} \mathrm{O}$ and extracted with EtOAc $(30 \mathrm{~mL} \times 3)$. The combined organic layer was dried over anhydrous $\mathrm{Na}_{2} \mathrm{SO}_{4}$, filtered, and concentrated. The crude product was purified by flash chromatography on silica gel eluting with Petroleum ether:DCM:EtOAc (2:1:2) to give 17a $\left(0.97 \mathrm{~g}\right.$, yield, 45\%). ${ }^{1} \mathrm{H}$ NMR (400 MHz, DMSO-d $d_{\text {б) }} \delta 8.60(\mathrm{~d}, J=2.4 \mathrm{~Hz}, 1 \mathrm{H}), 8.46(\mathrm{~d}, J=5.6 \mathrm{~Hz}, 1 \mathrm{H}), 8.17(\mathrm{~s}, 1 \mathrm{H}), 7.62(\mathrm{dd}$, $J=2.4,9.2 \mathrm{~Hz}, 1 \mathrm{H}), 7.42(\mathrm{dd}, J=4.8,8.8 \mathrm{~Hz}, 1 \mathrm{H}), 7.32(\mathrm{t}, J=8.8 \mathrm{~Hz}, 1 \mathrm{H}), 7.15(\mathrm{~d}, J$ $=8.8 \mathrm{~Hz}, 1 \mathrm{H}), 5.94(\mathrm{~s}, 1 \mathrm{H}), 5.82(\mathrm{q}, J=6.4 \mathrm{~Hz}, 1 \mathrm{H}), 1.67(\mathrm{~d}, J=7.4 \mathrm{~Hz}, 3 \mathrm{H})$. ESI-MS m/z: $431.4[\mathrm{M}+\mathrm{H}]^{+}$. 
To a solution of compund 16 (1.20 g, $0.005 \mathrm{~mol})$ and benzotriazol-1-yloxytris(dimethylamino)-phosphonium hexafluorophosphat (BOP) (2.90 g, $0.0065 \mathrm{~mol})$ in 20 $\mathrm{mL}$ acetonitrile, 1,8-Diazabicyclo[5.4.0]undec-7-ene (DBU) (1.1 mL, $0.0075 \mathrm{~mol})$ was added and the solution became clear. When precipitated solid was formed, the solution of 1-(2,6-Dichloro-3-fluorophenyl)-ethan-1-ol (1.56 g, $0.0075 \mathrm{~mol})$ and $\mathrm{NaH}$ ( $0.40 \mathrm{~g}, 60 \%$ dispersion in mineral oil) in $10 \mathrm{~mL}$ acetonitrile was added, and then stirred at room temperature overnight. The reaction mixture was poured into $100 \mathrm{~mL}$ $\mathrm{H}_{2} \mathrm{O}$ and extracted with EtOAc $(30 \mathrm{~mL} \times 3)$. The combined organic layer was dried over anhydrous $\mathrm{Na}_{2} \mathrm{SO}_{4}$, filtered, and concentrated. The crude product was purified by flash chromatography on silica gel eluting with petroleum ether:DCM:EtOAc $(2: 1: 2)$ to give 17b (1.2 g, yield, 55\%). ${ }^{1} \mathrm{H}$ NMR (400 MHz, $\left.\mathrm{CDCl}_{3}\right) \delta 8.23(\mathrm{~d}, J=2.0 \mathrm{~Hz}$, $1 \mathrm{H}), 7.70(\mathrm{dd}, J=2.4,9.2 \mathrm{~Hz}, 1 \mathrm{H}), 7.35(\mathrm{~d}, J=8.8 \mathrm{~Hz}, 1 \mathrm{H}), 7.27(\mathrm{dd}, J=5.2,8.8 \mathrm{~Hz}$, 1H), 7.05 (t, $J=8.4 \mathrm{~Hz}, 1 \mathrm{H}), 6.86(\mathrm{q}, J=6.8 \mathrm{~Hz}, 1 \mathrm{H}), 4.99(\mathrm{~s}, 2 \mathrm{H}), 1.91(\mathrm{~d}, J=6.9$ Hz, 3H). ESI-MS m/z: $432.4[\mathrm{M}+\mathrm{H}]^{+}$.

2-amino-N-(1-(2,6-dichloro-3-fluorophenyl)ethyl)-6-(1-methyl-1H-pyrazol-4-yl)quina zoline-4-amine (18)

Compound 18 was synthesized from 17a and 20 following the similar procedure described above for the preparation of 7a (yield, 51\%). mp 269-272 ${ }^{\circ} \mathrm{C} .{ }^{1} \mathrm{H}$ NMR (400 MHz, MeOD) $\delta 8.43(\mathrm{~d}, J=2.0 \mathrm{~Hz}, 1 \mathrm{H}), 8.01(\mathrm{~s}, 1 \mathrm{H}), 7.96(\mathrm{~s}, 1 \mathrm{H}), 7.81$ (dd, $J=1.6$, $8.4 \mathrm{~Hz}, 1 \mathrm{H}), 7.38-7.30(\mathrm{~m}, 2 \mathrm{H}), 7.14(\mathrm{t}, J=8.6 \mathrm{~Hz}, 1 \mathrm{H}), 6.04$ (q, $J=7.3 \mathrm{~Hz}, 1 \mathrm{H})$, $3.97(\mathrm{~s}, 3 \mathrm{H}), 1.80(\mathrm{~d}, J=7.2 \mathrm{~Hz}, 3 \mathrm{H}) .{ }^{13} \mathrm{C} \mathrm{NMR}(101 \mathrm{MHz}, \mathrm{MeOD}) \delta$ 159.6, 158.6, 155.9, 146.8, 139.9, 136.0, 130.7, 129.3, 127.6, 127.0, 122.5, 122.4, 118.3, 115.1, 114.9, 110.7, 48.9, 37.6, 16.2. ESI-MS m/z: 431.5 [M+H] $]^{+}$HRMS, ESI ${ }^{+}$m/z: Calcd for $\mathrm{C}_{20} \mathrm{H}_{17} \mathrm{Cl}_{2} \mathrm{FN}_{6}(\mathrm{M}+\mathrm{H})^{+}$, 431.0949; found, 431.0950 .

2-amino-N-(1-(2,6-dichloro-3-fluorophenyl)ethyl)-6-(1-(piperidin-4-yl)-1H-pyrazol-4 -yl)quinazoline-4-amine (19a)

Compound 19a was synthesized from 17a and 21 following the similar procedure described above for the preparation of $\mathbf{6}$ (yield, 74\%). mp $113-115{ }^{\circ} \mathrm{C} .{ }^{1} \mathrm{H}$ NMR (400 MHz, MeOD) $\delta 8.69$ (s, 1H), 8.24 (s, 1H), 8.07 - 8.03(m, 2H), 7.47 - 7.39 (m, 2H), $7.18(\mathrm{dd}, J=8.4,17.2 \mathrm{~Hz}, 1 \mathrm{H}), 6.09(\mathrm{q}, J=7.2 \mathrm{~Hz}, 1 \mathrm{H}), 4.65-4.63(\mathrm{~m}, 1 \mathrm{H})$, $3.64-3.53(\mathrm{~m}, 3 \mathrm{H}), 3.37(\mathrm{~s}, 1 \mathrm{H}), 3.28-3.17(\mathrm{~m}, 2 \mathrm{H}), 2.66(\mathrm{~d}, J=9.6 \mathrm{~Hz}, 1 \mathrm{H}), 2.40-$ $2.25(\mathrm{~m}, 5 \mathrm{H}), 1.84(\mathrm{~d}, J=7.2 \mathrm{~Hz}, 3 \mathrm{H}) .{ }^{13} \mathrm{C} \mathrm{NMR}(101 \mathrm{MHz}, \mathrm{MeOD}) \delta 138.8,137.2$, $136.6,132.4,129.9,129.7,127.9,125.8,121.2$, 119.3, 117.2, 115.6, 115.4, 114.3, 
110.0, 55.7, 49.8, 42.7, 42.6, 28.8, 28.6, 16.0. ESI-MS m/z: 500.5 [M+H] ${ }^{+}$. HRMS, $\mathrm{ESI}^{+}, \mathrm{m} / \mathrm{z}$ : Calcd for $\mathrm{C}_{24} \mathrm{H}_{24} \mathrm{Cl}_{2} \mathrm{FN}_{7}(\mathrm{M}+\mathrm{H})^{+}, 500.1527$; found, 500.1528 .

4-(1-(2,6-dichloro-3-fluorophenyl)ethoxy)-6-(1-(piperidin-4-yl)-1H-pyrazol-4-yl)quin azolin-2-amine (19b)

Compound 19b was synthesized from 17b and 21 following the similar procedure described above for the preparation of 6 (yield, 65\%). mp 267-269 ${ }^{\circ} \mathrm{C} .{ }^{1} \mathrm{H}$ NMR (400 MHz, DMSO- $\left.d_{6}\right) \delta 8.23(\mathrm{~s}, 1 \mathrm{H}), 8.06(\mathrm{~d}, J=2.0 \mathrm{~Hz}, 1 \mathrm{H}), 7.89-7.87(\mathrm{~m}$, $2 \mathrm{H}), 7.55(\mathrm{dd}, J=5.2,8.8 \mathrm{~Hz}, 1 \mathrm{H}), 7.44(\mathrm{t}, J=8.8 \mathrm{~Hz}, 1 \mathrm{H}), 7.34(\mathrm{~d}, J=8.8 \mathrm{~Hz}, 1 \mathrm{H})$, $6.94(\mathrm{q}, J=6.8 \mathrm{~Hz}, 1 \mathrm{H}), 6.39(\mathrm{~s}, 2 \mathrm{H}), 4.34-4.28(\mathrm{~m}, 1 \mathrm{H}), 3.16(\mathrm{~d}, J=14.4 \mathrm{~Hz}, 3 \mathrm{H})$, $2.76-2.70(\mathrm{~m}, 2 \mathrm{H}), 2.06-1.91(\mathrm{~m}, 4 \mathrm{H}), 1.85(\mathrm{~d}, J=7.2 \mathrm{~Hz}, 3 \mathrm{H}) .{ }^{13} \mathrm{C}$ NMR $(101$ MHz, MeOD) $\delta 166.2,159.5,158.6,151.2,137.5,136.3,132.0,129.9,128.9,126.8$, 125.3, 123.7, 122.2, 119.0, 116.2, 116.0, 111.5, 71.4, 56.1, 43.0, 29.4, 16.9. ESI-MS m/z: $501.4[\mathrm{M}+\mathrm{H}]^{+}$. HRMS, ESI ${ }^{+}, \mathrm{m} / \mathrm{z}$ : Calcd for $\mathrm{C}_{24} \mathrm{H}_{23} \mathrm{Cl}_{2} \mathrm{FN}_{6} \mathrm{O}(\mathrm{M}+\mathrm{H})^{+}, 501.1367$; found, 501.1369 .

\subsection{Kinase inhibitory activity}

The EGFR inhibitory activity was evaluated by using the Z'-LYTE technology platform (Life Technologies), and gefitinib was employed as the positive control. The experiments were performed according to the instructions of the manufacturer, and single point concentration testing $(1 \mu \mathrm{M})$ with two independent data points $(n=2)$. According to the antiproliferative activities and percent inhibition values of compounds, we chosed compounds $12 \mathrm{c}-12 \mathrm{e}$ and 13c-13d to evaluate kinase activity at ten concentration gradients from 1000 to $0.0508 \mathrm{nM}$. The $\mathrm{IC}_{50}$ values were calculated from the inhibition curves from two separate experiments.

\subsection{Cancer cell proliferation inhibition assay}

The antiproliferative activities of compounds were evaluated against MCF-7, A549, BT-474, SK-BR-3 and MDA-MB-231 cell lines by the standard MTT assay in vitro, with gefitinib as the positive control. All cell lines were purchased from Cell Bank of China Science Academy (Shanghai, China). All chemicals and solvents were purchased from Sigma-Aldrich or Gibco.

The BT-474 cell line was maintained in DEME medium supplement with $10 \%$ fetal bovine serum (FBS) and 1\% Penicillin-Streotomycin, others were cultured in RPMI 1640 medium aslo supplement with 10\% FBS and 1\% Penicillin-Streotomycin. 
Approximate $5 \times 10^{3}$ cells, suspended in medium, were plated into each well of a 96-well plate and grown at $37{ }^{\circ} \mathrm{C}$ in a humidified atmosphere with $5 \% \mathrm{CO}_{2}$ for $24 \mathrm{~h}$. The following day various concentrations of tested compounds were added to the culture medium and incubated for $72 \mathrm{~h}$. Fresh MTT was added to each well at the terminal concentration of $5 \mathrm{mg} / \mathrm{mL}$ in PBS, and incubated with cells at $37{ }^{\circ} \mathrm{C}$ for $4 \mathrm{~h}$. The formazan crystals in each well were dissolved in $150 \mu \mathrm{L}$ DMSO, and the absorbency at $570 \mathrm{~nm}$ was measured with an enzyme-linked immunosorbent assay plate reader. All of the compounds were tested three times in each of the cell lines.

\section{4 molecular modeling}

Molecular docking was performed with Surflex-Dock program that is interfaced with Sybyl 7.3 [23] according to our previously published protocol [24]. The crystal structure of EGFR kinase in complex with gefitinib (ID: 4WKQ) was obtained from protein data bank (PDB) [25]. All the water and ligands were removed and the random hydrogen atoms were added. The structures of the synthesized compounds were generated and minimized using tripos force fields. All the other default parameters were used except the factor was the threshold was set to 0.6 when the protomol was generated. The highest-scored conformation based on the Surflex-Dock scoring functions, was selected as the final bioactive conformation.

The MD simulations were performed using AMBER 12 software package [26, 27] according to our previously published protocol [28]. The missing residues, which were not solved in the crystal structures, were modeled using the loop building routine in Modeler [29]. Geometry optimization and the electrostatic potential calculations for gefitinib, 12c, 12e, and 7a were carried out at the HF/6-31G* level of the Gaussian03 suite [30]. The atomic partial charge was obtained by using the restrained electrostatic potential (RESP) fitting technique [31] implemented in AmberTools [32]. The force field parameters for gefitinib, 12c, 12e, and 7a were generated by the general amber force field (GAFF) [33] using the Antechamber program. The AMBER 99SB force field [34] was used to simulate the protein structure and the ionization state of amino acid residues were set according to the standard protocol. The model was neutralized by adding suitable counterions and were solvated in a truncated octahedron box of TIP3P [35] water molecules with a margin distance of $12 \AA$. The fully solvated and neutralized system was subjected to energy minimization. Following minimization, the system was gradually heated from 0 to $300 \mathrm{~K}$ in $50 \mathrm{ps}$ using a Langevin thermostat with a coupling coefficient of 1.0/ps with a force constant 2.0 
$\mathrm{kcal} \cdot \mathrm{mol}^{-1} \cdot \AA^{-2}$ on the complex. And then 50 ps of density equilibration with a force constant $2.0 \mathrm{kcal} \cdot \mathrm{mol}^{-1} \cdot \AA^{-2}$ on the complex were performed. Subsequently the systems were again equilibrated for 500 ps by releasing all the restrains. Finally, the $20 \mathrm{~ns}$ MD production was performed at $300 \mathrm{~K}$ with $1.0 \mathrm{~atm}$ pressure. During the MD simulations, the long-range Coulombic interactions were handled using the particle mesh Ewald (PME) method [36]. The cutoff distance for the long-range vdW energy term was set at $10.0 \AA$ A Periodic boundary conditions were applied to avoid edge effects in all calculations. The SHAKE algorithm [37] was employed on all atoms covalently bond to hydrogen atoms, allowing for an integration time step of 2 fs. Coordinate trajectories were recorded every 10 ps throughout all equilibration and production runs. The binding free energies $\left(\Delta \mathrm{G}_{\text {bind }}\right)$ were calculated by using MM-GBSA [38] procedure in AMBER12. Average 1000 snapshots were extracted from the last $10 \mathrm{~ns}$ MD trajectory for the calculations, and only 10 snapshots evenly extracted from the last $10 \mathrm{~ns}$ MD trajectory were used to calculate the entropic contribution.

\section{Acknowledgments}

This work was supported by grants from National Science Foundation of China (No. 815732630 and 81202413) and Guangdong Natural Science Foundation (No. 2015A030313285). The calculations of the ligands were performed in the China ScGrid of Supercomputing Center of Chinese Academy of Science.

\section{References}

[1] J. Mendelsohn, J. Baselga, The EGF receptor family as targets for cancer therapy, Oncogene. 19 (2000) 6550-6565.

[2] F. Ciardiello, G. Tortora, EGFR antagonists in cancer treatment, N Engl J Med. 358 (2008) $1160-1174$.

[3] A. Harandi, A.S. Zaidi, A.M. Stocker, D.A. Laber, Clinical efficacy and toxicity of anti-EGFR therapy in common cancers, Journal of oncology. 2009 (2009) 1-14.

[4] A. Gschwind, O.M. Fischer, A. Ullrich, The discovery of receptor tyrosine kinases: targets for cancer therapy, Nat Rrv Cancer. 4 (2004) 361-370.

[5] C. Carmi, A. Cavazzoni, S. Vezzosi, F. Bordi, F. Vacondio, C. Silva, S. Rivara, A. Lodola, R.R. Alfieri, S. La Monica, M. Galetti, A. Ardizzoni, P.G. Petronini, M. Mor, Novel irreversible epidermal growth factor receptor inhibitors by chemical modulation of the cysteine-trap portion, J. Med. Chem. 53 (2010) 2038-2050. 
[6] K. Singh, P. Sharma, A. Kumar, A. Chaudhary, R. Roy, 4-Aminoquinazoline analogs: a novel class of anticancer agents, Mini-Rev Med Chem. 13 (2013) 1177-1194.

[7] A.J. Barker, K.H. Gibson, W. Grundy, A.A. Godfrey, J.J. Barlow, M.P. Healy, J.R. Woodburn, S.E. Ashton, B.J. Curry, L.Scarlett, Studies leading to the identification of ZD1839 (Iressa ${ }^{\mathrm{TM}}$ ): an orally active, selective epidermal growth factor receptor tyrosine kinase inhibitor targeted to the treatment of cancer, Bioorg. Med. Chem. Lett. 11 (2001) 1911-1914.

[8] A.E. Wakeling, S.P. Guy, J.R. Woodburn, S.E. Ashton, B.J. Curry, A.J. Barker, K.H. Gibson, ZD1839 (Iressa) an orally active inhibitor of epidermal growth factor signaling with potential for cancer therapy, Cancer Res. 62 (2002) 5749-5754.

[9] E.R. Wood, A.T. Truesdale, O.B. McDonald, D. Yuan, A. Hassell, S.H. Dickerson, B. Ellis, C. Pennisi, E. Horne, K. Lackey, A unique structure for epidermal growth factor receptor bound to GW572016 (Lapatinib) relationships among protein conformation, inhibitor off-rate, and receptor activity in tumor cells, Cancer Res. 64 (2004) 6652-6659.

[10] K.N. Ganjoo, H. Wakelee, Review of erlotinib in the treatment of advanced non-small cell lung cancer, Biologics: targets \& therapy. 1 (2007) 335-346.

[11] Lee F. Allen, Irene A. Eiseman, David W. Fry, Peter F. Lenehan, CI-1033, an Irreversible pan-erbB Receptor Inhibitor and its Potential Application for the Treatment of Breast Cancer, Semin Oncol. 30 (2003) 65-78.

[12] E. Calvo, A.W. Tolcher, L.A. Hammond, A. Patnaik, J.S. de Bono, I.A. Eiseman, S.C. Olson, P.F. Lenehan, H. McCreery, P. LoRusso, Administration of CI-1033, an Irreversible Pan-erbB Tyrosine Kinase Inhibitor, Is Feasible on a 7-Day On, 7-Day Off Schedule A Phase I Pharmacokinetic and Food Effect Study, Clin Cancer Res. 10 (2004) 7112-7120.

[13] A. Gangjee, A. Vasudevan, S.F. Queener, R.L. Kisliuk, 6-Substituted 2, 4-Diamino-5-methyl-pyrido [2,3-d] pyrimidines as Inhibitors of Dihydrofolate Reductases from Pneumocystis carinii and Toxoplasma gondii and as Antitumor Agents, J. Med. Chem. 38 (1995) 1778-1785.

[14] G.W. Rewcastle, B.D. Palmer, A.M. Thompson, A.J. Bridges, D.R. Cody, H. Zhou, D.W. Fry, A. McMichael, W.A. Denny, Tyrosine kinase inhibitors. 10. Isomeric 4-[(3-bromophenyl) amino] pyrido [d]-pyrimidines are potent ATP binding site inhibitors of the tyrosine kinase function of the epidermal growth factor receptor, J. Med. Chem. 39 (1996) 1823-1835.

[15] D. Zhang, J. Ai, Z. Liang, C. Li, X. Peng, Y. Ji, H. Jiang, M. Geng, C. Luo, H. Liu, Discovery of novel 2-aminopyridine-3-carboxamides as c-Met kinase inhibitors, Bioorg. Med. Chem. 20 (2012) 5169-5180.

[16] W.M. Chen ,S.H. Wan, New Straightforward Synthesis of 2-Amino-6-methyl-5-(pyridin-4-ylsulfanyl)-3 H-quinazolin-4-one, Synthetic. Commun. 37 (2007) 53-61.

[17] BURNS Christopher john. BU Xianyong. WILKS Andrew Frederick, Protein kinase inhibitors, PCT Int. Appl. W03/099796, Cytopia Pty.Ltd, 2003.

[18] M. Campiglio, A. Locatelli, C. Olgiati, N. Normanno, G. Somenzi, L. Viganò, M. Fumagalli, S. Ménard, L. Gianni, Inhibition of proliferation and induction of apoptosis in breast cancer cells by 
the epidermal growth factor receptor (EGFR) tyrosine kinase inhibitor ZD1839 ('Iressa') is independent of EGFR expression level, J. Cell. Physiol. 198 (2004) 259-268.

[19] G.E. Konecny, M.D. Pegram, N. Venkatesan, R. Finn, G. Yang, M. Rahmeh, M. Untch, D.W. Rusnak, G. Spehar, R.J. Mullin, Activity of the dual kinase inhibitor lapatinib (GW572016) against HER-2-overexpressing and trastuzumab-treated breast cancer cells, Cancer Res. 66 (2006) 1630-1639.

[20] F.M. Sirotnak, M.F. Zakowski, V.A. Miller, H.I. Scher, M.G. Kris, Efficacy of cytotoxic agents against human tumor xenografts is markedly enhanced by coadministration of ZD1839 (Iressa), an inhibitor of EGFR tyrosine kinase, Clin Cancer Res. 6 (2000) 4885-4892.

[21] P. Warnault, A. Yasri, M. Coisy-Quivy, G. Cheve, C. Bories, B. Fauvel, R. Benhida, Recent advances in drug design of epidermal growth factor receptor inhibitors, Curr Med Chem. 20 (2013) 2043-2067.

[22] Y. Yang, Y. Shen, H. Liu, X. Yao, Molecular dynamics simulation and free energy calculation studies of the binding mechanism of allosteric inhibitors with p38a MAP kinase, J. Chem. Inf. Model. 51 (2011) 3235-3246.

[23] Sybyl 7.3, T.I., 1699 South Hanley Rd., St. Louis, MI 63144, USA.

[24] X. Wu, S. Wan, Z. Li, L. Yang, J. Zhang, S. Wu, 3D-QSAR study on 2, 3-dihydroimidazo [4, 5]-pyridin-2-one derivatives with a meta substitution pattern as V600EBRAF inhibitors, Medicinal Chemistry Research. 23 (2014) 587-602.

[25] http://www.rcsb.org/pdb/explore/explore.do?structureId=4WKQ.

[26] D.A. Case, T.A. Darden, T.E. Cheatham III, C.L. Simmerling, J. Wang, R.E. Duke, R. Luo, R.C. Walker, W. Zhang, K.M. Merz, B. Roberts, S. Hayik, A. Roitberg, G. Seabra, J. Swails, A.W. Goetz, I. Kolossváry, K.F. Wong, F. Paesani, J. Vanicek, R.M. Wolf, J. Liu, X. Wu, S.R. Brozell, T. Steinbrecher, H. Gohlke, Q. Cai, X. Ye, J. Wang, M.J. Hsieh, G. Cui, D.R. Roe, D.H. Mathews, M.G. Seetin, R. Salomon-Ferrer, C. Sagui, V. Babin, T. Luchko, S. Gusarov, A. Kovalenko, P.A. Kollman, AMBER 12, 2012, University of California, San Francisco.

[27] R. Salomon-Ferrer, A.W. Götz, D. Poole, S. Le Grand, R.C. Walker, Routine microsecond molecular dynamics simulations with AMBER on GPUs. 2. Explicit solvent particle mesh Ewald, J. Chem. Theory. Comput. 9 (2013) 3878-3888.

[28] X. Wu, S. Wan, G. Wang, H. Jin, Z. Li, Y. Tian, Z. Zhu, J. Zhang, Molecular dynamics simulation and free energy calculation studies of kinase inhibitors binding to active and inactive conformations of VEGFR-2, Journal of Molecular Graphics and Modelling. 56 (2015) 103-112.

[29] K. Arnold, L. Bordoli, J. Kopp, T. Schwede, The SWISS-MODEL workspace: a web-based environment for protein structure homology modelling, Bioinformatics. 22 (2006) 195-201.

[30] M. Frisch, G. Trucks, H. Schlegel, G. Scuseria, M. Robb, J. Cheeseman, J. Montgomery Jr, T. Vreven, K. Kudin, J. Burant. Gaussian 03; Gaussian, Inc.: Wallingford, CT, 2004.

[31] C.I. Bayly, P. Cieplak, W. Cornell, P.A. Kollman, A well-behaved electrostatic potential based method using charge restraints for deriving atomic charges: the RESP model, The Journal of 
Physical Chemistry. 97 (1993) 10269-10280.

[32] J. Wang, W. Wang, P.A. Kollman, D.A. Case, Automatic atom type and bond type perception in molecular mechanical calculations, J. Mol. Graph. Model. 25 (2006) 247-260.

[33] J. Wang, R.M. Wolf, J.W. Caldwell, P.A. Kollman ,D.A. Case, Development and testing of a general amber force field, J. Comput . Chem. 25 (2004) 1157-1174.

[34] V. Hornak, R. Abel, A. Okur, B. Strockbine, A. Roitberg, C. Simmerling, Comparison of multiple Amber force fields and development of improved protein backbone parameters, Proteins: Structure, Function, and Bioinformatics. 65 (2006) 712-725.

[35] W.L. Jorgensen, J. Chandrasekhar, J.D. Madura, R.W. Impey, M.L. Klein, Comparison of simple potential functions for simulating liquid water, J. Chem. Phys. 79 (1983) 926-935.

[36] T. Darden, D. York, L. Pedersen, Particle mesh Ewald: An N. $\log (\mathrm{N})$ method for Ewald sums in large systems, J. Chem. Phys. 98 (1993) 10089-10092.

[37] J.P. Ryckaert, G. Ciccotti ,H.J. Berendsen, Numerical integration of the cartesian equations of motion of a system with constraints: molecular dynamics of n-alkanes, J. Comput. Phys. 23 (1977) 327-341.

[38] P.A. Kollman, I. Massova, C. Reyes, B. Kuhn, S. Huo, L. Chong, M. Lee, T. Lee, Y. Duan ,W. Wang, Calculating structures and free energies of complex molecules: combining molecular mechanics and continuum models, Accounts. Chem. Res. 33 (2000) 889-897. 
Scheme 1. Reagents and conditions: (i) bromine, acetic acid, rt; (ii) formamide, reflux; (iii) $\mathrm{SOCl}_{2}$ and DMF, reflux; (iv) substituted-benzylamines, dioxane, rt; (v) $\mathrm{K}_{2} \mathrm{CO}_{3}, \mathrm{PdCl}_{2}(\mathrm{dppf}) \mathrm{CH}_{2} \mathrm{Cl}_{2}$, dioxane/ $\mathrm{H}_{2} \mathrm{O}$ (24:1), $85{ }^{\circ} \mathrm{C}$; (vi) $\mathrm{CH}_{2} \mathrm{Cl}_{2} / \mathrm{TFA}$ (4:1), rt.

Scheme 2. Reagents and conditions: (i) formamide, reflux; (ii) $\mathrm{SOCl}_{2}$ and DMF, reflux, (iii) for 11a-11b, substituted-benzylamines, $\mathrm{K}_{2} \mathrm{CO}_{3}$, dioxane, rt; for 11c, 1-(2,6-dichloro-3-fluorophenyl)ethanol, dioxane, $\mathrm{NaH}, 0{ }^{\circ} \mathrm{C}$-rt; for 11d-11f, substituted-anilines or 6-aminoquinoline, acetone, $\mathrm{HCl}$, reflux; for 11g, $\mathrm{Cs}_{2} \mathrm{CO}_{3}$, dioxane, rt; (iv) $\mathrm{K}_{2} \mathrm{CO}_{3}, \mathrm{PdCl}_{2}(\mathrm{dppf}) \mathrm{CH}_{2} \mathrm{Cl}_{2}$, dioxane/ $\mathrm{H}_{2} \mathrm{O}$ (24:1), $85{ }^{\circ} \mathrm{C}$; (v) $\mathrm{CH}_{2} \mathrm{Cl}_{2} / \mathrm{TFA}(4: 1)$, rt.

Scheme 3. Reagents and conditions: (i) dicyandiamide, sulfuric acid, $100{ }^{\circ} \mathrm{C}$; (ii) 4-Pyridinethiol, $\mathrm{KOH}$, ehylene glycol, $170{ }^{\circ} \mathrm{C}$; (iii) for 17a, BOP, DBU, 1-(2,6-Dichloro-3-fluorophenyl)ethan-1amine, acetonitrile, rt; for 17b, BOP, DBU, 1-(2,6-Dichloro-3-fluorophenyl)ethan-1-ol, NaH, acetonitrile, rt; (iv) $\mathrm{K}_{2} \mathrm{CO}_{3}, \mathrm{PdCl}_{2}$ (dppf) $\mathrm{CH}_{2} \mathrm{Cl}_{2}$, dioxane/ $\mathrm{H}_{2} \mathrm{O}$ (24:1), $85{ }^{\circ} \mathrm{C}$; (v) $\mathrm{CH}_{2} \mathrm{Cl}_{2} / \mathrm{TFA}$ (4:1), rt.

Fig. 1. Structure of EGFR small molecule inhibitors.

Fig. 2. Preliminary structure-activity relationships.

Fig. 3. Conformation comparison between redocked (cyan) and the co-crystallized (green) gefitinib binding to EGFR.

Fig.4. Binding mode between EGFR kinase and reprentative compounds. (gefitinib in stick and ball model, 7a in green stick model, 12c in blue stick model, and 19a in red stick model).

Fig. 5. RMSDs of backbone atoms $(\mathrm{C}, \mathrm{C} \alpha$, and $\mathrm{N})$ of the systems against the initial structures.

Fig. 6. Binding mode comparison between redocked (green) and MD simulated representative snapshots (cyan) of 12c (left panel) and 12e (right panel) in the active site of EGFR. Yellow dots represent hydrogen bonds. (EGFR in colored cartoon, ligands in stick model, the key residues in lines model). 
Table 1. The kinase inhibitory activities and in vitro antiproliferative effects of synthesized compounds.<smiles>[R7]c1ncnc2ncc(-c3cnn([R])c3)cc12</smiles>

6, 7a-7d<smiles>[R7]c1ncnc2ccc(-c3cnn([R])c3)cc12</smiles>

12a-12f, 13a-13d<smiles>[R]c1nc(N)nc2ccc(-c3cnn([R])c3)cc12</smiles>

$18,19 a-19 b$

\begin{tabular}{|c|c|c|c|c|c|c|c|c|c|}
\hline \multirow{2}{*}{ Com } & \multirow{2}{*}{$\mathrm{X}$} & \multirow{2}{*}{$\mathrm{R}_{1}$} & \multirow{2}{*}{$\mathrm{R}_{2}$} & \multirow{2}{*}{$\begin{array}{c}\text { EGFR } \\
\text { inhibition at } \\
1 \mu \mathrm{M}(\%)^{\mathrm{a}}\end{array}$} & \multicolumn{5}{|c|}{ In vitro antiproliferative effects $\left(\mathrm{IC}_{50}, \mu \mathrm{M}\right)^{\mathrm{b}}$} \\
\hline & & & & & MCF-7 & A549 & BT-474 & SK-BR-3 & MDA-MB-231 \\
\hline 6 & NH & & & 17 & 1.63 & 29.73 & 13.47 & 17.67 & 7.11 \\
\hline $7 \mathbf{a}$ & NH & & $\mathrm{CH}_{3}$ & 9 & $>100$ & $>100$ & $>100$ & $>100$ & $>100$ \\
\hline $7 b$ & NH & & $\mathrm{CH}_{3}$ & 0 & $>100$ & $>100$ & $>100$ & $>100$ & $>100$ \\
\hline $7 c$ & NH & & $\mathrm{CH}_{3}$ & 18 & $>100$ & $>100$ & $>100$ & $>100$ & $>100$ \\
\hline $7 d$ & $\mathrm{NH}$ & & $\mathrm{CH}_{3}$ & 30 & $>100$ & $>100$ & $>100$ & $>100$ & $>100$ \\
\hline $12 a$ & NH & & $\mathrm{CH}_{3}$ & 67 & 48.90 & 30.40 & 35.95 & 28.49 & 48.90 \\
\hline $12 b$ & $\mathrm{O}$ & & $\mathrm{CH}_{3}$ & 11 & 22.55 & 28.79 & 45.60 & 27.20 & 30 \\
\hline $12 c$ & $\mathrm{NH}$ & & $\mathrm{CH}_{3}$ & 101 & 52.20 & $>100$ & 5.26 & 3.10 & 62.65 \\
\hline $12 d$ & $\mathrm{NH}$ & & $\mathrm{CH}_{3}$ & 101 & 0.49 & 1.38 & 10.93 & 0.064 & 26.28 \\
\hline $12 \mathrm{e}$ & $\mathrm{NH}$ & & $\mathrm{CH}_{3}$ & 102 & 11.37 & 9.86 & 5.68 & 5.87 & 16.93 \\
\hline $12 f$ & $\mathrm{O}$ & & $\mathrm{CH}_{3}$ & 43 & 21.97 & 26.63 & 24.30 & 14.42 & 16.51 \\
\hline
\end{tabular}




196

${ }^{\mathrm{a}}$ Values are the average of two independent experiments.

${ }^{\mathrm{b}}$ The data were means from at least three independent experiments.

Table 2. In vitro enzymatic inhibitory activities of compounds $\mathbf{1 2 c - 1 2 e}$ and $\mathbf{1 3 c - 1 3 d}$ against different types of EGFR.

\begin{tabular}{cccccc}
\hline \multirow{2}{*}{ Com } & \multicolumn{5}{c}{${\text { EGFR }\left(\mathrm{IC}_{50}, \mathrm{nM}\right)^{\mathrm{a}}}^{\mathrm{a}}$} \\
\cline { 2 - 6 } & $\begin{array}{c}\text { EGFR } \\
\text { (ErbB1) }\end{array}$ & $\begin{array}{c}\text { EGFR (ErbB1) } \\
\text { L858R }\end{array}$ & $\begin{array}{c}\text { EGFR (ErbB1) } \\
\text { L858R /T790M }\end{array}$ & $\begin{array}{c}\text { ErbB2 } \\
(\text { HER2) }\end{array}$ & $\begin{array}{c}\text { ErbB4 } \\
(\text { HER4) }\end{array}$ \\
\hline 12c & 2.97 & 3.50 & $>1000$ & 676 & 801 \\
$\mathbf{1 2 d}$ & 44.1 & 108 & $>1000$ & 874 & 930 \\
12e & 3.58 & 4.25 & $>1000$ & 590 & 782 \\
$\mathbf{1 3 c}$ & 31.9 & 39.1 & $>1000$ & 1202 & 1603 \\
13d & 28.2 & 35.0 & $>1000$ & 1350 & 1100 \\
gefitinib & 0.585 & 0.637 & $1013^{[21]}$ & $1830^{[21]}$ & ND $^{\mathrm{b}}$ \\
\hline
\end{tabular}

${ }^{\mathrm{a}}$ Values are the average of two independent experiments.

${ }^{\mathrm{b}} \mathrm{ND}$, not determined. 
Table 3 Hydrogen Bonds analysis from MD

\begin{tabular}{cccccc}
\hline & Donor & Acceptor & Distance $(\AA)^{\mathrm{a}}$ & Angle $\left.^{\left({ }^{\circ}\right.}\right)^{\mathrm{b}}$ & ${\text { Occupancy }(\%)^{\mathrm{c}}}$ \\
\hline Gefitinib-EGFR & Met 793 NH & Ligand N & $3.163(0.15)$ & $24.38(11.31)$ & 92.45 \\
7a- EGFR & Met 793 NH & Ligand N & $3.291(0.16)$ & $34.78(15.22)$ & 0.95 \\
12c-EGFR & Met 793 NH & Ligand N & $3.118(0.15)$ & $22.17(12.34)$ & 95.55 \\
12e-EGFR & Met 793 NH & Ligand N & $3.141(0.15)$ & $26.30(13.19)$ & 89.65 \\
\hline
\end{tabular}

${ }^{\mathrm{a}}$ The average distance with standard error $\left(\mathrm{SE}=\right.$ standard deviation $\left./ \mathrm{N}^{1 / 2}\right)$ in parentheses between hydrogen-acceptor atom and hydrogen-donor atom in the investigated time period. ${ }^{\mathrm{b}}$ The average angle with standard error ( $\mathrm{SE}=$ standard deviation $/ \mathrm{N}^{1 / 2}$ ) in parentheses for hydrogen bond in the investigated time period. ${ }^{\mathrm{c}}$ Occupancy is in unit of percentage of the investigated time period.

Table 4 MM-GBSA binding free energies and its components for the studied complexes for the last $10 \mathrm{~ns}$ MD trajectories ${ }^{\mathrm{a}}$

\begin{tabular}{ccccccccc}
\hline & $\Delta \mathrm{G}_{\mathrm{vdW}}$ & $\Delta \mathrm{G}_{\text {ele }}$ & $\Delta \mathrm{G}_{\mathrm{MM}}$ & $\Delta \mathrm{G}_{\text {elesol }}$ & $\Delta \mathrm{G}_{\mathrm{np}, \text { sol }}$ & $\Delta \mathrm{H}_{\text {pred }}$ & $-\mathrm{T} \Delta \mathrm{S}$ & $\Delta \mathrm{G}_{\text {pred }}$ \\
\hline \multirow{2}{*}{ gefitinib } & -47.78 & -90.05 & -137.83 & 108.48 & -6.17 & -35.52 & 22.15 & \multirow{2}{*}{ 7a } \\
& $(0.10)$ & $(0.40)$ & $(0.43)$ & $(0.41)$ & $(0.01)$ & $(0.10)$ & $(2.94)$ & -13.37 \\
& -41.51 & -7.55 & -49.06 & 25.89 & -5.22 & -28.39 & 18.33 & \\
\multirow{2}{*}{ 12c } & $(0.07)$ & $(0.08)$ & $(0.13)$ & $(0.10)$ & $(0.01)$ & $(0.07)$ & $(2.30)$ & -10.06 \\
& -44.38 & -11.42 & -55.81 & 25.23 & -5.51 & -36.08 & 22.31 & \\
\multirow{2}{*}{ 12e } & $-4.08)$ & $(0.11)$ & $(0.14)$ & $(0.11)$ & $(0.01)$ & $(0.08)$ & $(0.61)$ & -13.77 \\
& $-0.09)$ & $(0.08)$ & $(0.13)$ & $(0.08)$ & $(0.01)$ & $(0.09)$ & $(1.36)$ & \\
\hline
\end{tabular}

${ }^{\mathrm{a}}$ Mean energies are in $\mathrm{kcal} / \mathrm{mol}$, with corresponding standard errors $\left(\mathrm{SE}=\right.$ standard deviation $\left./ \mathrm{N}^{1 / 2}\right)$ in parentheses. $\Delta \mathrm{H}$ : the enthalpy changes, $\Delta \mathrm{H}=\Delta \mathrm{G}_{\text {ele }}+\Delta \mathrm{G}_{\mathrm{vdW}}+\Delta \mathrm{G}_{\mathrm{np}, \mathrm{sol}}+\Delta \mathrm{G}_{\mathrm{ele}, \text { sol }}$. $\mathrm{T} \Delta \mathrm{S}$ : the entropy changes. $\Delta \mathrm{G}_{\text {pred }}$ : the calculated binding free energy by MM-GBSA method. 


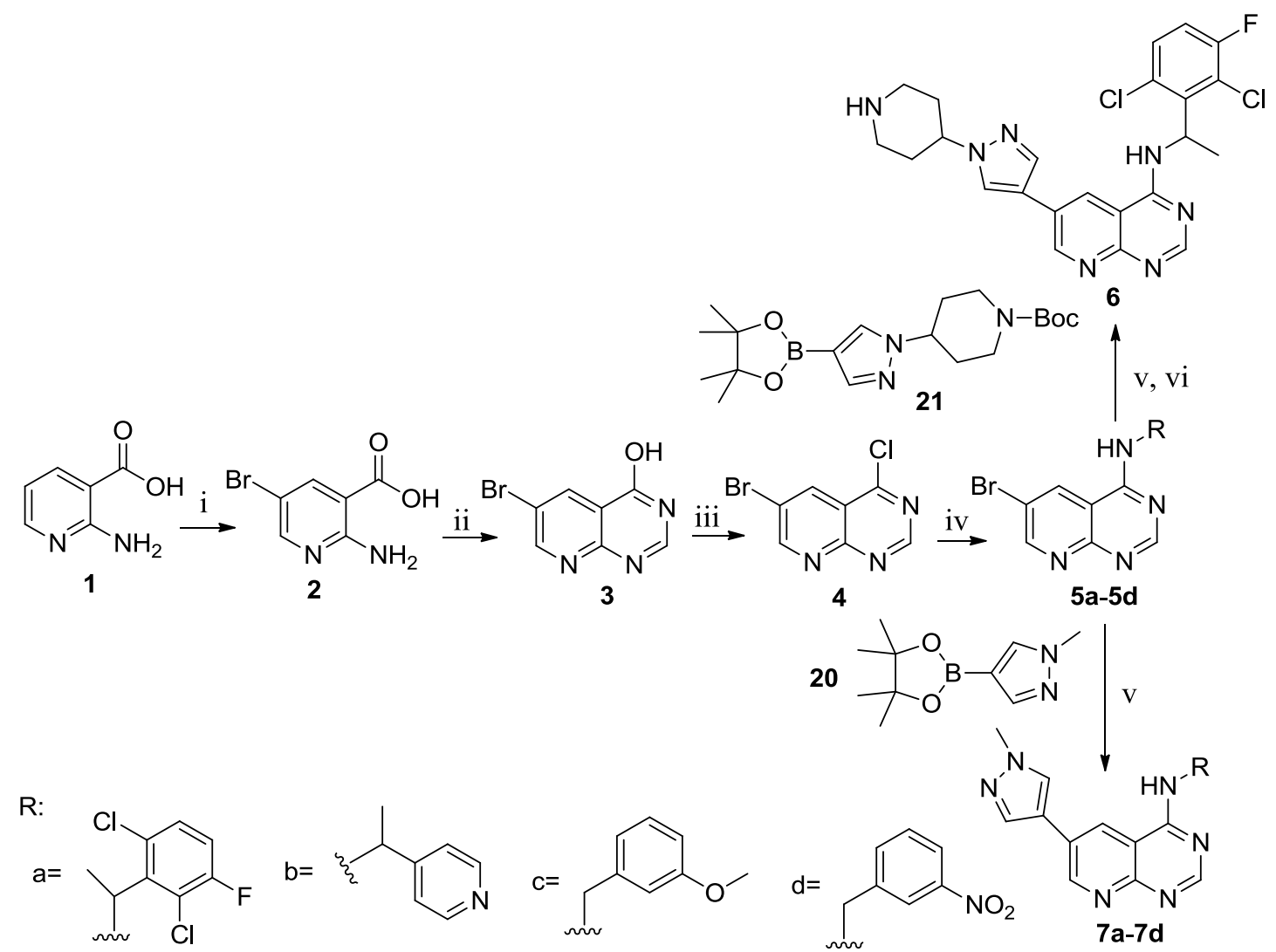

Scheme 1. Reagents and conditions: (i) bromine, acetic acid, rt; (ii) formamide, reflux; (iii) $\mathrm{SOCl}_{2}$ and DMF, reflux; (iv) substituted-benzylamines, dioxane, rt; (v) $\mathrm{K}_{2} \mathrm{CO}_{3}, \mathrm{PdCl}_{2}(\mathrm{dppf}) \mathrm{CH}_{2} \mathrm{Cl}_{2}$, dioxane $/ \mathrm{H}_{2} \mathrm{O}$ (24:1), $85^{\circ} \mathrm{C}$; (vi) $\mathrm{CH}_{2} \mathrm{Cl}_{2} / \mathrm{TFA}$ (4:1), rt. 
<smiles>Nc1ccc(Br)cc1C(=O)O</smiles><smiles>[R]c1ncnc2ccc(-c3cnn(C)c3)cc12</smiles><smiles>Oc1ncnc2ccc(Br)cc12</smiles>

12a-12f<smiles>Cn1cc(B2OC(C)(C)C(C)(C)C2(C)C)cn1</smiles>
20<smiles>[R][X]c1ncnc2cc(Br)ccc12</smiles><smiles>CC(C)(C)OC(=O)N1CCC(n2cc(B3OC(C)(C)C(C)(C)O3)cn2)CC1</smiles><smiles>[R][X]c1ncnc2ccc(-c3cnn(C4CCNCC4)c3)cc12</smiles>

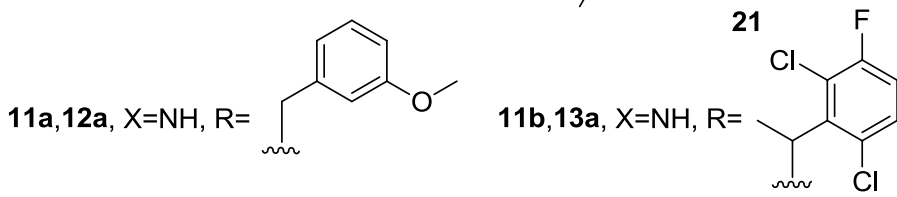

11c, $12 b, 13 b, X=O, R=$<smiles>CC(C)c1c(Cl)ccc(F)c1Cl</smiles>

11d,12c,13c, $X=\mathrm{NH}, \mathrm{R}=$<smiles>Fc1ccc(I)cc1Cl</smiles>
$11 e, 12 d, 13 d, X=N H, R=$<smiles>Cc1ccc2ncccc2c1</smiles>
11f, 12e, $X=N H, R=$<smiles>C#Cc1cccc(CF)c1</smiles>

11g, 12f, $X=O, R=$<smiles>[3H]Cc1ccc2ncccc2c1</smiles>

Scheme 2. Reagents and conditions: (i) formamide, reflux; (ii) $\mathrm{SOCl}_{2}$ and DMF, reflux, (iii) for 11a-11b, substituted-benzylamines, $\mathrm{K}_{2} \mathrm{CO}_{3}$, dioxane, $\mathrm{rt}$; for 11c, 1-(2,6-dichloro-3-fluorophenyl)ethanol, dioxane, $\mathrm{NaH}, 0{ }^{\circ} \mathrm{C}-\mathrm{rt}$; for 11d-11f, substituted-anilines or 6 -aminoquinoline, acetone, $\mathrm{HCl}$, reflux; for 11g, $\mathrm{Cs}_{2} \mathrm{CO}_{3}$, dioxane, rt; (iv) $\mathrm{K}_{2} \mathrm{CO}_{3}, \mathrm{PdCl}_{2}$ (dppf) $\mathrm{CH}_{2} \mathrm{Cl}_{2}$, dioxane/ $\mathrm{H}_{2} \mathrm{O}$ (24:1), $85^{\circ} \mathrm{C}$; (v) $\mathrm{CH}_{2} \mathrm{Cl}_{2} / \mathrm{TFA}(4: 1)$, rt.

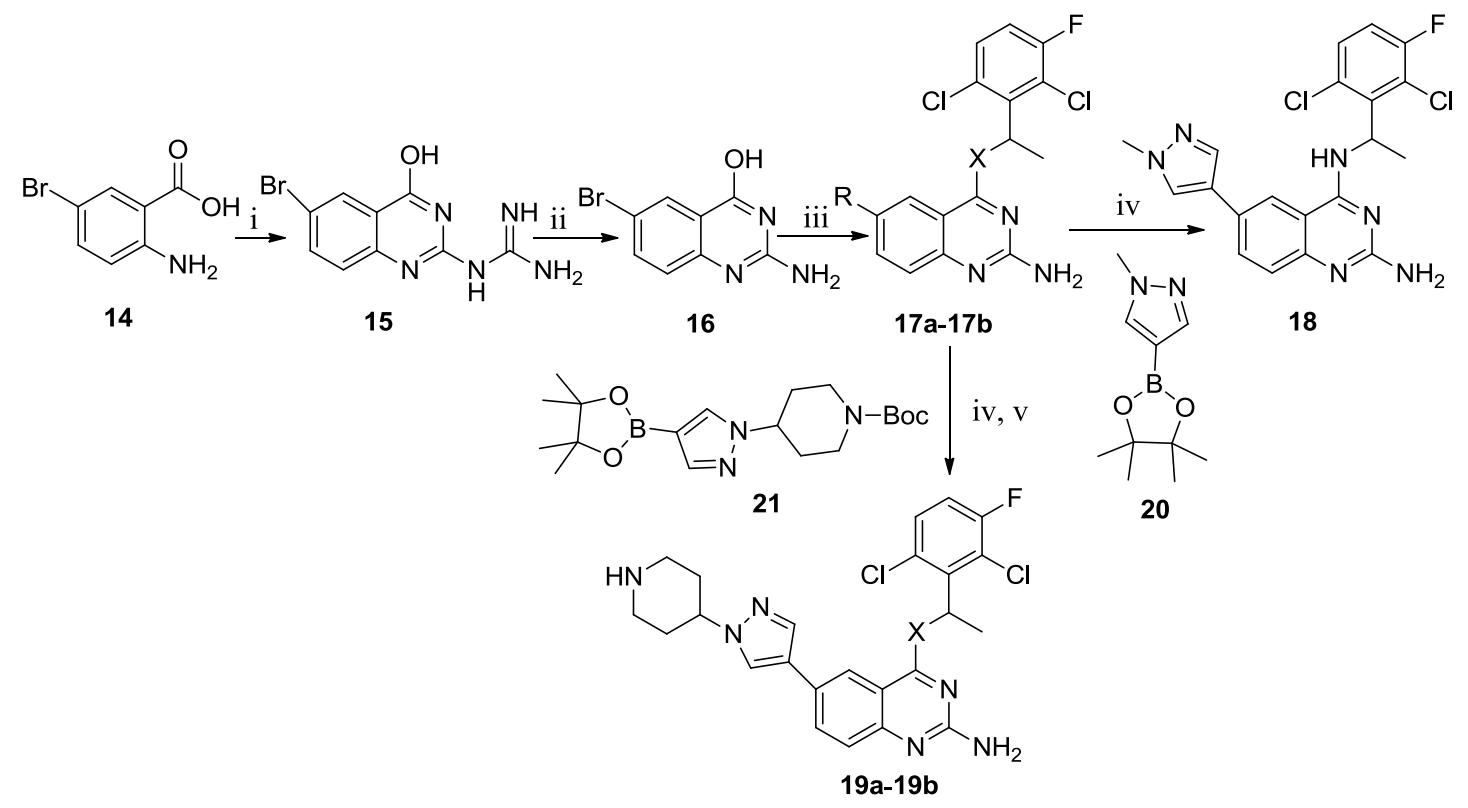

17a, 19a: $X=N H ; 17 b, 19 b: X=O$ 
Scheme 3. Reagents and conditions: (i) dicyandiamide, sulfuric acid, $100{ }^{\circ} \mathrm{C}$; (ii) 4-Pyridinethiol, $\mathrm{KOH}$, ehylene glycol, $170^{\circ} \mathrm{C}$; (iii) for 17a, BOP, DBU, 1-(2,6-Dichloro-3-fluorophenyl)ethan-1amine, acetonitrile, rt; for 17b, BOP, DBU, 1-(2,6-Dichloro-3-fluorophenyl)ethan-1-ol, NaH, acetonitrile, rt; (iv) $\mathrm{K}_{2} \mathrm{CO}_{3}, \mathrm{PdCl}_{2}$ (dppf) $\mathrm{CH}_{2} \mathrm{Cl}_{2}$, dioxane/ $\mathrm{H}_{2} \mathrm{O}$ (24:1), $85^{\circ} \mathrm{C}$; (v) $\mathrm{CH}_{2} \mathrm{Cl}_{2} / \mathrm{TFA}$ (4:1), rt. 
<smiles>COc1cc2c(Nc3ccc(F)c(Cl)c3)ncnc2cc1OCCCN1CCOCC1</smiles>

gefitinib

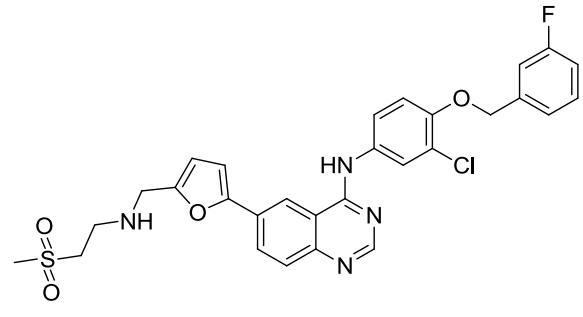

lapatinib

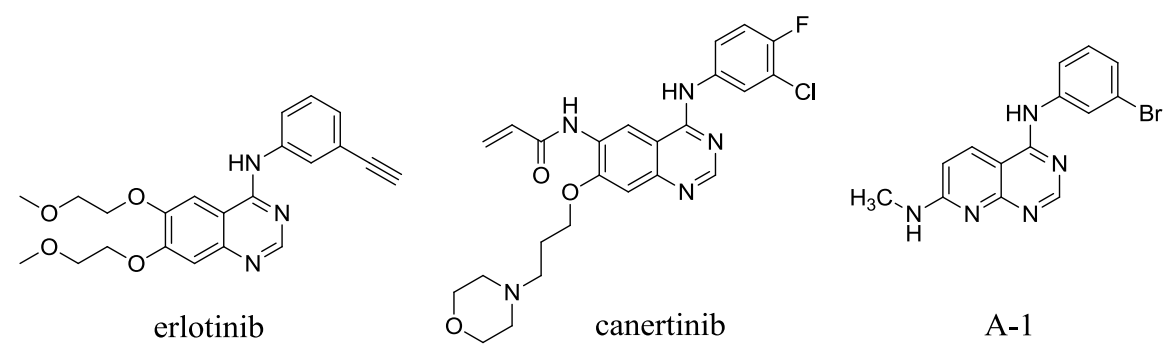

Fig. 1. Structure of EGFR small molecule inhibitors. (1.5-column fitting image)

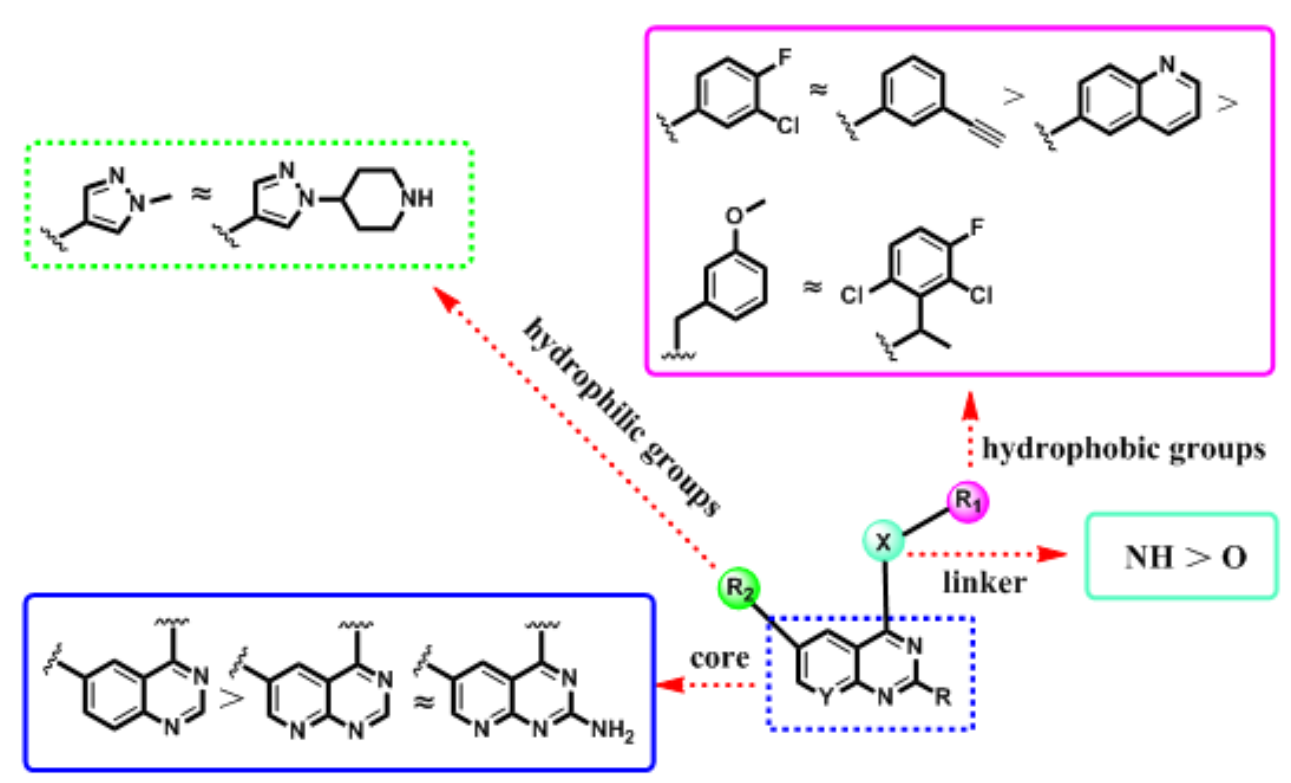

Fig. 2. Preliminary structure-activity relationships. (1.5-column fitting image) 


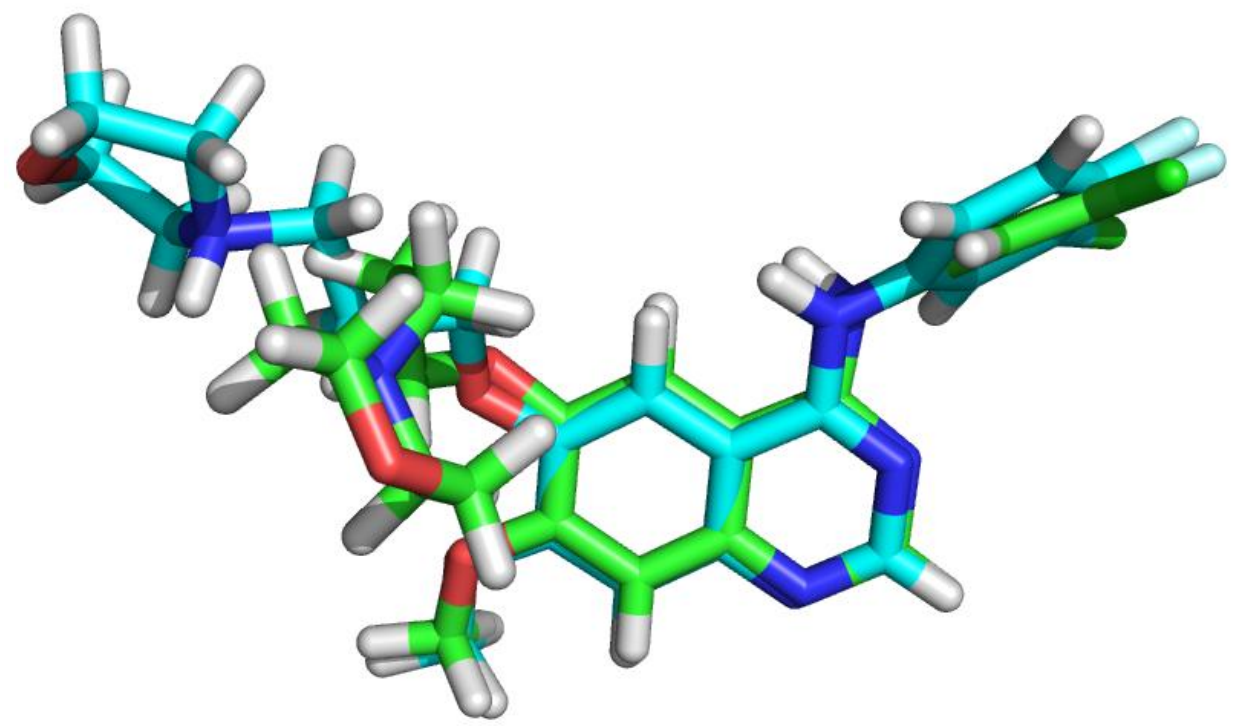

Fig. 3. Conformation comparison between redocked (cyan) and the co-crystallized (green) gefitinib binding to EGFR. (1.5-column fitting image)

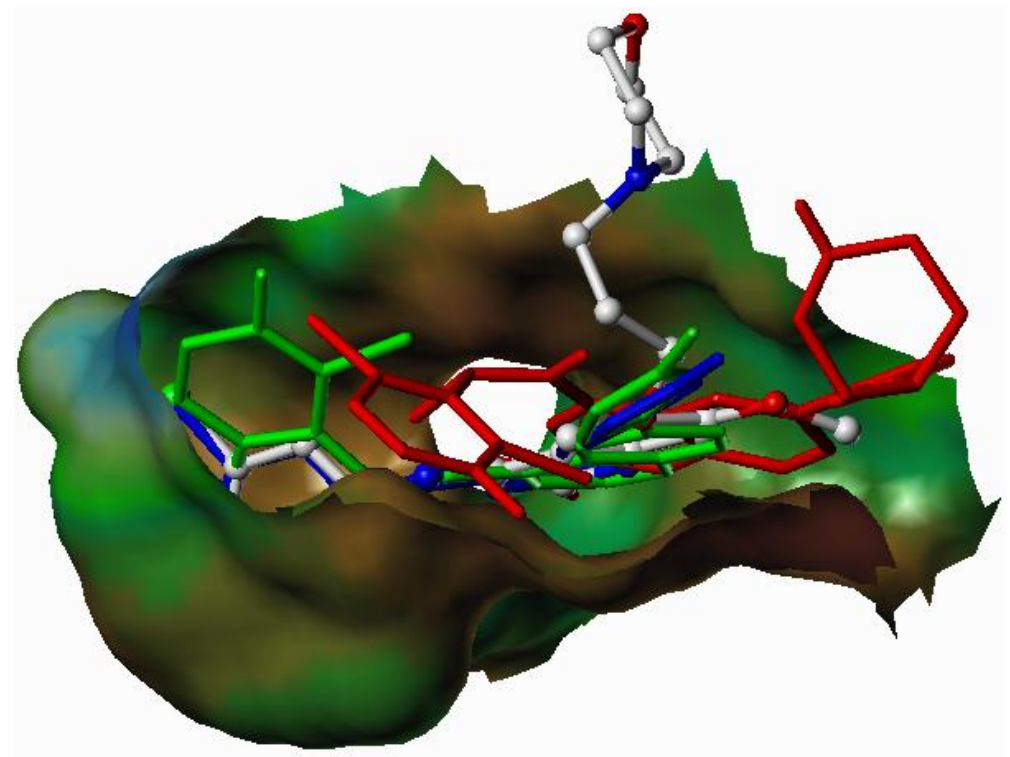

Fig.4. Binding mode between EGFR kinase and reprentative compounds. (gefitinib in stick and ball model, 7a in green stick model, 12c in blue stick model, and 19a in red stick model). (1.5-column fitting image) 


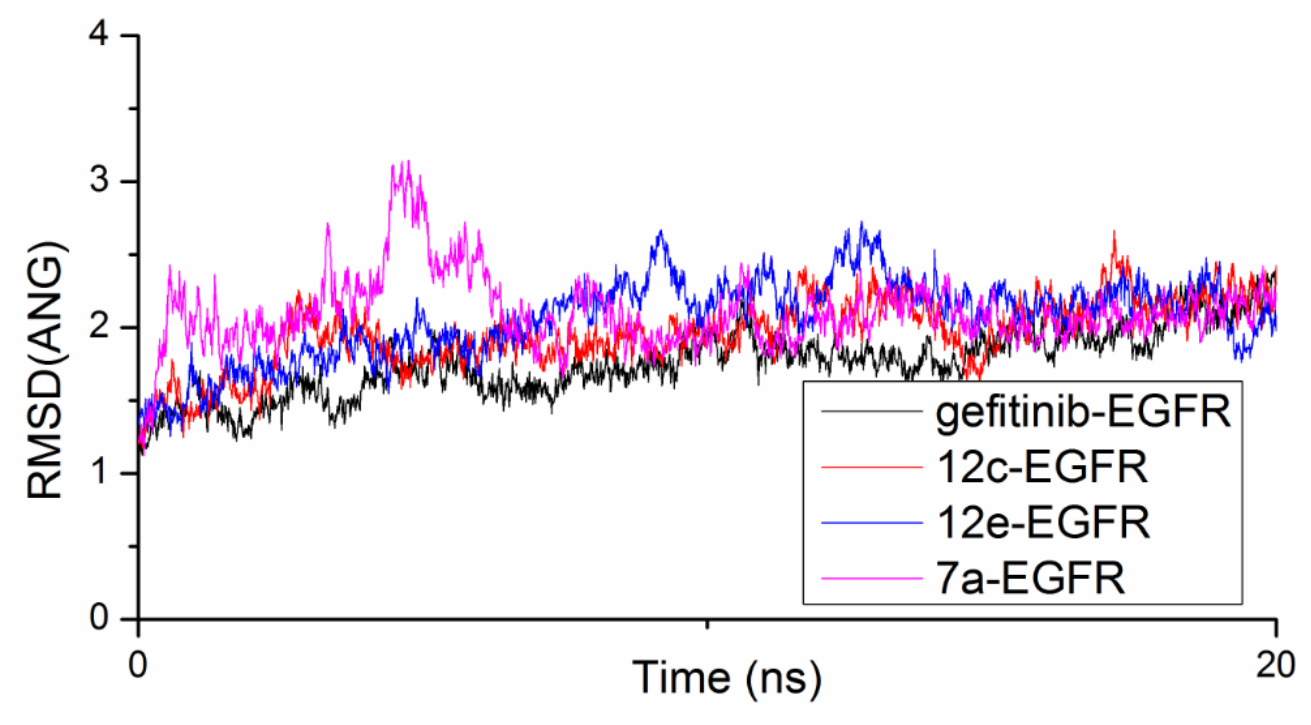

Fig. 5. RMSDs of backbone atoms $(C, C \alpha$, and $N)$ of the systems against the initial structures.

(1.5-column fitting image )
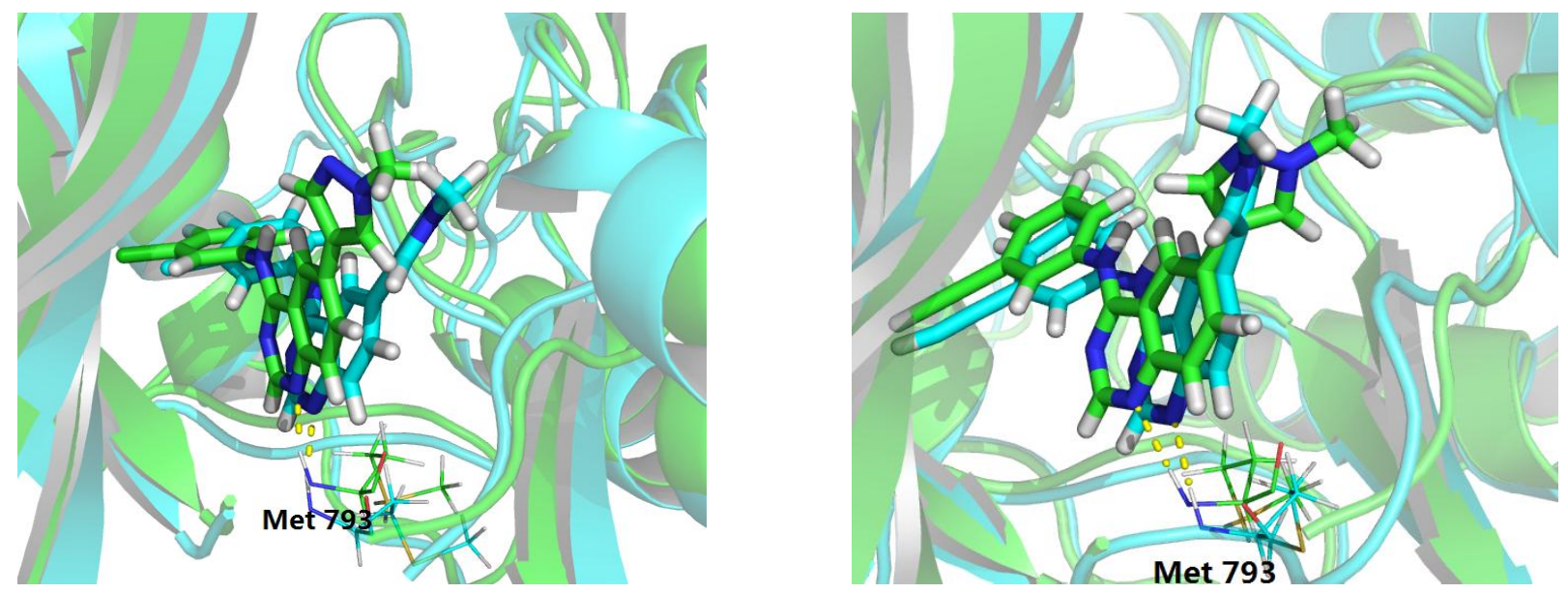

Fig. 6. Binding mode comparison between redocked (green) and MD simulated representative snapshots (cyan) of 12c (left panel) and 12e (right panel) in the active site of EGFR. Yellow dots represent hydrogen bonds. (EGFR in colored cartoon, ligands in stick model, the key residues in lines model).(2-column fitting image) 
Compounds 12c and 12e displayed remarkably higher EGFR inhibitory activity $\left(\mathrm{IC}_{50}=2.97 \mathrm{nM}\right.$ and $3.58 \mathrm{nM}$, respectively $)$ and good anti-proliferative effect against SK-BR-3 cell $\left(\mathrm{IC}_{50}=3.10 \mu \mathrm{M}\right.$ and $5.87 \mu \mathrm{M}$, respectively).<smiles>Cn1cc(-c2ccc3ncnc(Nc4ccc(F)c(Cl)c4)c3c2)cn1</smiles>

$12 \mathrm{c}$<smiles>C#Cc1cccc(Nc2ncnc3ccc(-c4cnn(C)c4)cc23)c1</smiles>

$12 \mathrm{e}$
EGFR IC $_{50}=2.97 \mathrm{nM}$ SK-BR-3 IC $_{50}=3.10 \mu \mathrm{M}$
EGFR IC $_{50}=3.58 \mathrm{nM}$

SK-BR-3 $\mathrm{IC}_{50}=5.87 \mu \mathrm{M}$ 